University of Windsor

Scholarship at UWindsor

1991

\title{
A unified treatment of the non-relativistic and relativistic hydrogen atom II: The Green functions
}

\author{
R. A. Swainson \\ Gordon W. F. Drake \\ University of Windsor
}

Follow this and additional works at: https://scholar.uwindsor.ca/physicspub

Part of the Physics Commons

\section{Recommended Citation}

Swainson, R. A. and Drake, Gordon W. F.. (1991). A unified treatment of the non-relativistic and relativistic hydrogen atom II: The Green functions. Journal of Physics A: Mathematical and General, 24 (1), 95-120. https://scholar.uwindsor.ca/physicspub/35 
A unified treatment of the non-relativistic and relativistic hydrogen atom II: the Green functions

This article has been downloaded from IOPscience. Please scroll down to see the full text article.

1991 J. Phys. A: Math. Gen. 2495

(http://iopscience.iop.org/0305-4470/24/1/020)

View the table of contents for this issue, or go to the journal homepage for more

Download details:

IP Address: 137.207.184.30

The article was downloaded on 26/04/2013 at 02:42

Please note that terms and conditions apply. 


\title{
A unified treatment of the non-relativistic and relativistic hydrogen atom II: the Green functions
}

\author{
Robin A Swainson and G W F Drake \\ Department of Physics, University of Windsor, Windsor, Ontario, Canada N9B 3P4
}

Received 26 June 1990

\begin{abstract}
This is the second in a series of three papers in which it is shown how the radial part of non-relativistic and relativistic hydrogenic bound-state calculations involving the Green functions can be presented in a unified manner. In this paper the non-relativistic Green function is examined in detail; new functional forms are presented and a clear mathematical progression is shown to link these and most other known forms. A linear transformation of the four radial parts of the relativistic Green function is given which allows for the presentation of this function as a simple generalization of the non-relativistic Green function. Thus, many properties of the non-relativistic Green function are shown to have simple relativistic generalizations. In particular, new recursion relations of the radial parts of both the non-relativistic and relativistic Green functions are presented, along with new expressions for the double Laplace transforms and recursion relations between the radial matrix elements. A novel proof of the Sturmian form of the radial Green functions is given in an appendix.
\end{abstract}

\section{Introduction}

This is the second in a series of three papers in which we present a unified treatment of non-relativistic and relativistic calculations of the properties of hydrogen-like systems involving the Coulomb Green functions (CGF). In the first paper [1] we presented a simple linear transformation which uncouples the pair of first-order differential equations defining the radial Dirac-Coulomb wave functions. We showed how this leads us to two second-order differential equations for the transformed radial wavefunctions which are generalizations of the differential equation satisfied by the radial Schrödinger-Coulomb wavefunction, and which are therefore immediately soluble.

In the present paper we extend this work to the analysis of the general Coulomb Green functions. Indeed, we show how an extension of the linear transformation allows us to exhibit the relativistic Dirac-Coulomb Green function (DCGF) as a generalization of the non-relativistic Schrödinger-Coulomb Green function (SCGF). It follows that much of the analysis required for specific calculations of non-relativistic and relativistic effects, such as matrix elements, double Laplace transforms, recursion relations and so on, can be treated in a unified manner. In the following paper [2] we will extend this work further to consider the reduced Coulomb Green functions (RCGF), functions appearing in bound-state perturbation theory.

As we remarked in the first paper of this series [1] powerful methods [3] are currently available for the treatment of the angular part of problems in the atomic physics of hydrogen-like systems. Our work then, focuses mainly on the radial part of the analysis, and in that respect differs from other attempts to unify non-relativistic 
and relativistic calculations [1]. Furthermore we have confined ourselves to the analysis required for calculations involving bound states-the extension to unbound states will be presented elsewhere.

Knowledge of the general CGF is required for the study of various atomic properties of hydrogen-like systems. Two-photon bound-state transitions and the Lamb shift are but two examples [4]. Even second-order perturbation calculations, though ostensib!y requiring the use of the RCGF, may actually involve the general CGF treated in this paper. The polarizability of hydrogenic ions is a case in point. Selection rules arising from the angular part of the calculation lead to integrals of the general CGF and not the RCGF as will be shown in a further paper devoted to a study of that effect.

Since the remainder of this paper will be concerned with a rather detailed and specific analysis of the radial CGFs, it is probably worthwhile to present a brief review of the theory of Green functions in a more abstract setting. Given a Hermitian operator $H$, the corresponding resolvent or Green operator $G(z)$ is defined by

$$
(H-z) G(z)=1
$$

where $z$, referred to later on as the 'energy variable' is a complex number. Usually $H$ will have associated with it a complete set of eigenfunctions $\psi_{E}$ corresponding generally to both discrete and continuous eigenvalues $E$, so that

$$
\begin{aligned}
& (H-E) \dot{\psi}_{E}=0 \\
& \Sigma \psi_{E} \psi_{E}^{\dagger}=1
\end{aligned}
$$

It should now be clear that a formal expression for $G(z)$ is given by

$$
G(z)=\Sigma \psi_{E} \psi_{E}^{+} /(E-z)
$$

If $H$ is represented by a differential operator $H_{r}$ acting on a Hilbert space of functions on $\mathbb{R}^{3}, G(z)$ is itself represented by a function $G\left(r_{1}, r_{2} ; z\right)$ on $\mathbb{R}^{3} \times \mathbb{R}^{3}$ which satisfies

$$
\left(H_{r_{1}}-z\right) G\left(\boldsymbol{r}_{1}, \boldsymbol{r}_{2} ; z\right)=\delta\left(\boldsymbol{r}_{1}-\boldsymbol{r}_{2}\right) \text {. }
$$

We shall be concerned here exclusively with the Schrödinger- and Dirac-Coulomb Green functions. In view of the nature of the Hamiltonians involved, we see that the former is a scalar function, while the latter is a $4 \times 4$ matrix-valued function.

The SCGF is defined and its various forms reviewed in section 2 . There has been a number of methods used to determine its functional form, ranging from the direct computation of the sum in (1.4), to expansions as sums of products of radial functions and angular functions, solutions in parabolic coordinates, solutions in momentum space, and solutions in phase space.

It appears that the first published attempt to calculate the SCGF was by Meixner in 1933 [5] where he only partially succeeded in solving the three-dimensional problem in parabolic coordinates. Following that, a solution of the radial functions was discovered involving the product of a homogeneous Whittaker function with an inhomogeneous one. Integral representations for the full Green function in coordinate space were not found until the 1960's, and were given in papers by Wichmann and Woo [6] and by Hostler [7]. The full Green function in momentum space had been derived in the late 1940's by Schwinger [8], who made use of the explicit character of the $S O(4)$ symmetry of the non-relativistic hydrogen atom when it is represented in momentum space. This work was published somewhat later though. Hostler [7] also derived an integral representation for the radial function which has since been frequently used by several authors. In 1970 Hostler [9], while examining the Coulomb 
Green function in $n$-dimensional space, came across what is now referred to as the Sturmian form of the radial Green function and which is basically an infinite series of Laguerre polynomials. In the context of the phase-space formulation of the theory of the hydrogen atom, Garcia-Bondia [10] and later Chetouani and Hamman [11] derived forms for the full Green function, the latter authors giving it in terms of parabolic coordinates.

In section 2 we present a self-contained analysis of both the radial and full SCGF in coordinate space. The Sturmian form of the radial function is explicitly derived directly from the defining differential equation in appendix 1 . We show how it is possible to derive many other forms for the Green function, appealing only to familiar properties of the various special functions involved. Thus a clear line of mathematical reasoning is established between the Sturmian form and all others.

In section 3 we turn our attention to the DCGF. As in the non-relativistic case, there have been several different approaches to the discovery of suitable functional forms. These have been obviously less successful; the $\mathrm{SO}(4)$ symmetry is broken in the relativistic Kepler problem. Most popular has been the solution in terms of a partial wave expansion.

Apparently the first derivation of the radial DCGF was made by Wichmann and Kroll in 1956 [12] in connection with a study of vacuum polarization effects in hydrogenic ions. Their solution, written in terms of Whittaker functions, has become the standard form. Shortly thereafter, as we explained in the previous paper [1], Martin and Glauber [13] were able to derive the full DCGF using a method based on the second-order Dirac equation. The DCGF is obtained from the Green function corresponding to this equation by the application of a projection operator. Attempts at a Sturmian form, which appeared somewhat later in the 1970's, were also based on the Green function of the second-order Dirac equation [14]. Other authoss [15] have reported the derivation of forms for the full Green function, but these seem to have little applicability. Common to all of these methods is the rather complicated nature of the solution. Since the standard solutions to the radial Dirac-Cculomb equation involve generally two different terms, the corresponding Green functions contain four terms. This causes difficulties when computing matrix elements.

In section 3 we present a transformation of the defining radial equations for the DCGF which allows for the formulation of the relativistic problem in a manner quite analogous to the non-relativistic problem and which gives simple one-term solutions. The method allows us to use the analysis of appendix 1 and gives the radial function in Sturmian form; other forms can be obtained in precisely the same manner as is used for the radial SCGF. As we pointed out in the first paper in this series [1], the approach we take is closely related to the method of solving the Dirac equation first noted by Infeld [16]. Furthermore, in contrast to the second-order theories, which are three-dimensional, our ap proach retains the standard angular analysis and concentrates on simplifying the radial part of the problem.

In section 4 we present raising and lowering operators for the radial CGFs, which will be seen to be analogous to the angular momentum raising and lowering operators of the radial wavefunctions. These give rise to relationships between different radial CGFs which are of intriñsic intererest in themsetves, althought they also lead tô rèlationships between the matrix elements of different CGFs. Of course the solution of any particular problem in atomic physics using the CGF generally requires the calculation of matrix elements. In section 5 we give general forms for the double Laplace transforms (DLT) of the radial CGFs from which any matrix element can be derived. Sometimes, 
relationships exist between different matrix elements of the same CGF. In section 6 we show how a generalization of the hypervirial theorem, well known in the study of matrix elements of hydrogenic wavefunctions, can lead to such relationships.

\section{Review of the Schrödinger-Coulomb Green function}

Several different techniques have been employed by various authors in an effort to derive useful functional forms for the non-relativistic hydrogenic Green function [4]. The technique we present here is quite direct. We explicitly solve the defining differential equation, and derive further forms using widely known properties of the special functions. The Schrödinger-Coulomb Green function (SCGF) $G\left(\boldsymbol{r}_{1}, \boldsymbol{r}_{2} ; E\right)$ corresponding to the energy variable $E$ is the solution of

$$
\left[-\frac{\hbar^{2}}{2 m} \nabla_{1}^{2}-\frac{\hbar^{2}}{a m r_{1}}-E\right] G\left(\boldsymbol{r}_{1}, \boldsymbol{r}_{2} ; E\right)=\delta\left(\boldsymbol{r}_{1}-\boldsymbol{r}_{2}\right)
$$

subject to suitable boundary conditions. (The scaled Bohr radius $a$ is related to the Bohr radius $a_{0}$ by $a=a_{0} / Z$.) Expanding $G$ in terms of spherical harmonics so that [17]:

$$
G\left(r_{1}, r_{2} ; E\right)=\sum_{l m} g_{l}\left(r_{1}, r_{2} ; E\right) Y_{l m}\left(\theta_{1}, \phi_{1}\right) Y_{l m}^{*}\left(\theta_{2}, \phi_{2}\right) .
$$

and noting also that

$$
\delta\left(r_{1}-r_{2}\right)=\frac{\delta\left(r_{1}-r_{2}\right)}{r_{1} r_{2}} \sum_{l m} Y_{l m}\left(\theta_{1}, \phi_{1}\right) Y_{l m}^{*}\left(\theta_{2}, \phi_{2}\right)
$$

leads us to the defining equation for the radial part of the SCGF,

$\left[\frac{1}{r_{1}^{2}} \frac{\mathrm{d}}{\mathrm{d} r_{1}}\left(r_{1}^{2} \frac{\mathrm{d}}{\mathrm{d} r_{1}}\right)-\frac{l(l+1)}{r_{1}^{2}}+\frac{2}{a r_{1}}-\frac{1}{v^{2} a^{2}}\right] g_{l}\left(r_{1}, r_{2} ; \nu\right)=-\frac{2 m}{\hbar^{2}} \frac{\delta\left(r_{1}-r_{2}\right)}{r_{1} r_{2}}$

where we have written $\nu^{2} a^{2}=-\hbar^{2} / 2 m E$. The boundary conditions we impose on $g_{l}$ are:

$$
\lim _{r_{1} \rightarrow 0} r_{1} g_{l}\left(r_{1}, r_{2}\right)=0 \quad r_{1} g_{l} \in L^{2}(\mathbb{R}) .
$$

In appendix 1 we have solved a slightly more general equation than (2.4) using a modified version of the method we presented in the previous paper [1] for solving the defining equation of the radial wavefunctions. We actually solve the Laplace transform of our generalized equation, which is of first order and relatively simple to deal with. In the next section we will see how the solutions of our generalized equation are also applicable to the solution of the relativistic problem. Applied here to the non-relativistic problem, the solution of the generalized equation (A1.3) leads to the so-called Sturmian form [9] of the radial SCGF:

$$
\begin{aligned}
g_{l}\left(r_{1}, r_{2} ; \nu\right)= & \left(2 m / \hbar^{2}\right) g_{\nu l}\left(r_{1}, r_{2} ; 1 / a \nu\right) \\
= & \left(2 m / \hbar^{2}\right)(2 / a \nu)^{2 l+1}\left(r_{1} r_{2}\right)^{l} \mathrm{e}^{-\left(r_{1}+r_{2}\right) / a \nu} \\
& \times \sum_{k=0}^{\infty} \frac{k !}{(2 l+1+k) !(l+1+k-\nu)} L_{k}^{2 l+1}\left(\frac{2 r_{1}}{a \nu}\right) L_{k}^{2 l+1}\left(\frac{2 r_{2}}{a \nu}\right) .
\end{aligned}
$$

Notice the poles at $n \equiv \nu=I+1+k$ corresponding to the energy eigenvalues $E_{n}=$ $-(\alpha Z)^{2} m c^{2} / 2 n^{2}$, with $l=0, \ldots, n-1$. Thus we have obtained, en passant, the discrete 
part of the hydrogenic spectrum. It is often remarked that the SCGF can be expressed as a sum over the discrete spectrum alone. We see it as a consequence of our solution of the defining differential equation.

We now proceed to derive several additional forms which we will find useful when calculating the matrix elements of the SCGF. We begin with the following representation of the modified Bessel function, $I_{\nu}[18]$,

$$
\begin{gathered}
\left(\frac{2 r_{1}}{a \nu} \frac{2 r_{2}}{a \nu} t\right)^{-1-1 / 2}(1-t)^{-1} \exp \left(-\frac{t}{1-t} \frac{2}{a \nu}\left(r_{1}+r_{2}\right)\right) I_{2 l+1}\left(\frac{4 \sqrt{r_{1} r_{2} t}}{a \nu(1-t)}\right) \\
=\sum_{k=0}^{\infty} \frac{k !}{(2 l+1+k) !} t^{k} L_{k}^{2 l+1}\left(\frac{2 r_{1}}{a \nu}\right) L_{k}^{2 l+1}\left(\frac{2 r_{2}}{a \nu}\right)
\end{gathered}
$$

together with

$$
\int_{0}^{1} t^{t+k-\nu} \mathrm{d} t=(l+k+1-\nu)^{-1}
$$

to arrive at our first variant:

$$
\begin{aligned}
g_{l}\left(r_{1}, r_{2} ; \nu\right)= & \left(2 m / \hbar^{2}\right) \frac{1}{\sqrt{r_{1} r_{2}}} \\
& \times \int_{0}^{1} t^{-\nu-\frac{1}{2}}(1-t)^{-1} \exp \left(-\frac{\left(r_{1}+r_{2}\right)(1+t)}{a \nu(1-t)}\right) I_{2 t+1}\left(\frac{4 \sqrt{r_{1} r_{2} t}}{a \nu(1-t)}\right) \mathrm{d} t .
\end{aligned}
$$

Now we make a change of variables $t \mapsto s=1-t$ and find

$$
\begin{aligned}
g_{l}\left(r_{1}, r_{2} ; \nu\right)= & \left(2 m / \hbar^{2}\right) \frac{1}{\sqrt{r_{1} r_{2}}} \mathrm{e}^{\left(r_{1}+r_{2}\right) / a \nu} \\
& \times \int_{0}^{1}(1-s)^{-\nu-\frac{1}{2} s^{-1}} \exp \left(-\frac{2\left(r_{1}+r_{2}\right)}{a \nu s}\right) I_{2 l+1}\left(\frac{4 \sqrt{r_{1} r_{2}(1-s)}}{a \nu s}\right) \mathrm{d} s
\end{aligned}
$$

which is useful for calculating double Laplace transforms of the radial Green functions (see section 4 and [19]).

As a final change of variable of integration we let $s \mapsto \rho=\cosh ^{-1}(2 / s-1)$ to arrive at a form of $g_{l}$ which has been frequently used by other authors [7]:

$$
\begin{aligned}
g_{l}\left(r_{1}, r_{2} ; \nu\right)= & \left(2 m / \hbar^{2}\right) \frac{1}{\sqrt{r_{1} r_{2}}} \int_{0}^{\infty}\left(\operatorname{coth} \frac{\rho}{2}\right)^{2 \nu} \exp \left(-\frac{\left(r_{1}+r_{2}\right)}{a \nu} \cosh \rho\right) \\
& \times I_{2 l+1}\left(\frac{2 \sqrt{r_{1} r_{2}}}{a \nu} \sinh \rho\right) \mathrm{d} \rho .
\end{aligned}
$$

We can now write down the 'standard' representation of the radial SCGF in terms of regular and irregular Whittaker functions, $M$ and $W$ (see appendix 4 ) by noting that [20]

$$
\begin{aligned}
W_{\nu, \mu / 2}\left(a_{1} t\right) & M_{\nu, \mu / 2}\left(a_{2} t\right) \\
= & \frac{t \sqrt{a_{1} a_{2}} \Gamma(\mu+1)}{\Gamma(1 / 2+\mu / 2-\nu)} \int_{0}^{\infty}\left(\operatorname{coth} \frac{\rho}{2}\right)^{2 \nu} \\
& \quad \times \exp \left(-\frac{\left(a_{1}+a_{2}\right)}{2} t \cosh \rho\right) I_{\mu}\left(t \sqrt{a_{1} a_{2}} \sinh \rho\right) \mathrm{d} \rho
\end{aligned}
$$


(for $\left.\mathscr{R}(1 / 2+\mu / 2-\eta)>0, \mathscr{R}(t)>0, a_{1}>a_{2}\right)$ from which follows [7]

$$
g_{l}\left(r_{1}, r_{2} ; \nu\right)=\frac{m a \nu}{\hbar^{2}} \frac{\Gamma(l+1-\nu)}{(2 l+1) !} \frac{1}{r_{1} r_{2}} M_{\nu, l+2}\left(\frac{2 r_{\varepsilon}}{a \nu}\right) W_{\nu, l+1}\left(\frac{2 r_{2}}{a \nu}\right)
$$

where $r_{<}=\min \left\{r_{1}, r_{2}\right\}$ and $r_{>}=\max \left\{r_{1}, r_{2}\right\}$.

Further integral representations are possible. Returning to equation (2.6) and noting that [18]

$$
\mathrm{e}^{-z} z^{\alpha / 2} L_{n}^{\alpha}(z)=\frac{1}{n !} \int_{0}^{\infty} \mathrm{e}^{-s} s^{n+\alpha / 2} J_{\alpha x}(2 \sqrt{s z}) \mathrm{d} s
$$

where $n+\mathscr{R}(\alpha)>-1$ and $J_{\alpha}$ is an ordinary Bessel function, we find a new double integral formula for $g_{l}$ :

$$
\begin{aligned}
g_{l}\left(r_{1}, r_{2} ; \nu\right)= & \left(2 m / \hbar^{2}\right) \frac{\mathrm{e}^{\left(r_{1}+r_{2}\right) / a \nu}}{\sqrt{r_{1} r_{2}}} \int_{0}^{\infty} \int_{0}^{\infty} \mathrm{e}^{-s_{1}-s_{2}} J_{2 l+1}\left(2 \sqrt{\frac{2 r_{1} s_{1}}{a \nu}}\right) \\
& \times J_{2 l+1}\left(2 \sqrt{\frac{2 r_{2} s_{2}}{a \nu}}\right) \sum_{k=0}^{\infty} \frac{\left(s_{1} s_{2}\right)^{k+l+1} \frac{1}{(2 l+1+k) ! k !(l+1+k-\nu)}}{\left(l+s_{1} \mathrm{~d} s_{2} .\right.} .
\end{aligned}
$$

The sum in the last equation can be expressed as an integral over a modified Bessel function using equation (2.8),

$$
\sum_{k=0}^{\infty} \frac{\left(s_{1} s_{2}\right)^{k+l+\frac{1}{2}}}{(2 l+1+k) ! k !(l+1+k-\nu)}=\int_{0}^{\infty} t^{-\nu-\frac{1}{2}} I_{2 l+1}\left(2 \sqrt{s_{1} s_{2} t}\right) d t
$$

and this leads us to our final form for the radial SCGF, a new triple integral representation:

$$
\begin{aligned}
g_{1}\left(r_{1}, r_{2} ; \nu\right)= & \left(2 m / \hbar^{2}\right) \frac{\mathrm{e}^{\left(r_{1}+r_{2}\right) / a \nu}}{\sqrt{r_{1} r_{2}}} \int_{0}^{\infty} \int_{0}^{\infty} \mathrm{e}^{-s_{1}-s_{2}} J_{2 l+1}\left(2 \sqrt{\frac{2 r_{1} s_{1}}{a \nu}}\right) \\
& \times J_{2 t+1}\left(2 \sqrt{\frac{2 r_{2} s_{2}}{a \nu}}\right) \int_{0}^{\infty} t^{-\nu-\frac{1}{2}} I_{2 l+1}\left(2 \sqrt{s_{2} s_{2} t}\right) \mathrm{d} t \mathrm{~d} s_{1} \mathrm{~d} s_{2} .
\end{aligned}
$$

It is interesting that by performing the integrals over $s_{1}$ and $s_{2}$ in $(2.17)$ we arrive at equation (2.9).

We now take a step backwards. From (2.2)

$$
\begin{aligned}
G\left(\boldsymbol{r}_{1}, \boldsymbol{r}_{2} ; E\right) & =\sum_{l m} g_{l}\left(r_{1}, r_{2} ; \nu\right) Y_{l m}\left(\theta_{1}, \phi_{1}\right) Y_{l m}^{*}\left(\theta_{2}, \phi_{2}\right) \\
& =\sum_{l} g_{l}\left(r_{1}, r_{2} ; \nu\right)(2 l+1) P_{l}\left(\hat{\boldsymbol{r}}_{1} \cdot \hat{\boldsymbol{r}}_{2}\right)
\end{aligned}
$$

where $P_{l}$ is a Legendre polynomial of degree $l$. In view of (2.10) we can write

$$
\begin{aligned}
G\left(r_{1}, r_{2} ; E\right)= & \left(2 m / \hbar^{2}\right) 1 / \sqrt{r_{1} r_{2}} \int_{0}^{\infty}\left(\operatorname{coth} \frac{\rho}{2}\right)^{2 \nu} \exp \left(-\frac{\left(r_{1}+r_{2}\right)}{a \nu} \cosh \rho\right) \\
& \times \sum_{l}(2 l+1) P_{l}\left(\hat{r}_{1} \cdot \hat{r}_{2}\right) I_{2 l+1}\left(\frac{2 \sqrt{r_{1} r_{2}}}{a \nu} \sinh \rho\right) \mathrm{d} \rho
\end{aligned}
$$

and, since

$$
\begin{aligned}
& \rho / 2 I_{0}\left(\rho \sqrt{(1+\tau) / 2)}=\sum_{l}(2 l+1) P_{l}(\tau) I_{2 l+1}(\rho)\right. \\
& G\left(\boldsymbol{r}_{1}, \boldsymbol{r}_{2} ; E\right)=\left(2 m / \hbar^{2} a \nu\right) \int_{0}^{\infty}\left(\operatorname{coth} \frac{\rho}{2}\right)^{2 \prime} \sinh \rho \exp \left(-\frac{\left(r_{1}+r_{2}\right)}{a \nu} \cosh \rho\right) \\
& \times I_{0}\left(\frac{\sinh \rho}{a \nu} \sqrt{2 r_{1} r_{2}}\left(1+\hat{r}_{1} \cdot \hat{r}_{2}\right)\right) \mathrm{d} \rho .
\end{aligned}
$$


This form was first presented by Hostler [7]. We can of course resort to a variable we have used previously: $\rho \mapsto t$ where $\cosh \rho=(1+t) /(1-t)$. Then $(2.20)$ becomes

$$
\begin{aligned}
G\left(\boldsymbol{r}_{1}, \boldsymbol{r}_{2} ; E\right)= & \left(2 m / \hbar^{2} a \nu\right) \int_{0}^{\infty} \frac{2 t^{-\nu}}{(1-t)^{2}} \exp \left(-\frac{\left(r_{1}+r_{2}\right)}{a \nu}\left(\frac{1+t}{1-t}\right)\right) \\
& \times I_{0}\left(\frac{2\left[2 t r_{1} r_{2}\left(1+\hat{\boldsymbol{r}}_{1} \cdot \hat{\boldsymbol{r}}_{2}\right)\right]^{1 / 2}}{a \nu(1-t)}\right) \mathrm{d} t .
\end{aligned}
$$

We are now in a position to find a 'Sturmian' expansion of the SCGF in parabolic coordinates. Recalling the definition of this coordinate system,

$$
x=\sqrt{\xi \eta} \cos \varphi \quad y=\sqrt{\xi \eta} \sin \varphi \quad z=(\xi-\eta) / 2
$$

it is not difficult to show that

$$
2 r_{1} r_{2}\left(1+\hat{r}_{1} \cdot \hat{r}_{2}\right)=\xi_{1} \xi_{2}+\eta_{1} \eta_{2}+2 \sqrt{\xi_{1} \xi_{2} \eta_{1} \eta_{2}} \cos \left(\varphi_{2}-\varphi_{1}\right)
$$

We also note that $[18]$

$$
I_{0}\left(\sqrt{a^{2}+b^{2}+2 a b \cos \theta}\right)=\sum_{m=-\infty}^{+\infty}(-1)^{m} \mathrm{e}^{\mathrm{i} m \theta} I_{m}(a) I_{m}(b) .
$$

Thus, from (2.21) we can write

$$
G\left(\boldsymbol{r}_{1}, \boldsymbol{r}_{2} ; E\right)=\sum_{-\infty}^{+\infty}(-1)^{m} \mathrm{e}^{\mathrm{i} m\left(\varphi_{1}-\varphi_{2}\right)} g_{m}\left(\xi_{1}, \eta_{1} ; \xi_{2}, \eta_{2} ; \nu\right)
$$

where [11]

$$
\begin{aligned}
g_{m}\left(\xi_{1}, \eta_{1} ; \xi_{2},\right. & \left.\eta_{2} ; \nu\right) \\
= & \left(4 m / \hbar^{2} a \nu\right) \int_{0}^{\infty} \frac{t^{-\nu}}{(1-t)^{2}} \exp \left[\frac{-\left(\xi_{1}+\eta_{1}+\xi_{2}+\eta_{2}\right)}{2 a \nu}\left(\frac{1+t}{1-t}\right)\right] \\
& \times I_{m}\left(\frac{2 \sqrt{t \xi_{1} \xi_{2}}}{a \nu(1-t)}\right) I_{m}\left(\frac{2 \sqrt{t \eta_{1} \eta_{2}}}{a \nu(1-t)}\right) \mathrm{d} t .
\end{aligned}
$$

Now, as in (2.7)

$$
\begin{aligned}
I_{m}\left(\frac{2 \sqrt{t \xi_{1} \xi_{2}}}{a \nu(1-t)}\right) & \\
= & (1-t) \exp \left(\frac{t\left(\xi_{1}+\xi_{2}\right)}{a \nu(1-t)}\right)\left(\frac{t \xi_{1} \xi_{2}}{a^{2} \nu^{2}}\right)^{|m| / 2} \sum_{k=0}^{\infty} \frac{k ! t^{k}}{(k+|m|) !} \\
& \times L_{k}^{|m|}\left(\xi_{1} / a \nu\right) L_{k}^{|m|}\left(\xi_{2} / a \nu\right)
\end{aligned}
$$

since $I_{m}=I_{-m}$. Thus we arrive at the following form of the Green function in parabolic coordinates:

$$
\begin{aligned}
g_{m}\left(\xi_{1}, \eta_{1} ; \xi_{2},\right. & \left.\eta_{2} ; \nu\right) \\
= & \frac{\left(4 m / \hbar^{2}\right)}{(a \nu)^{2|m|+1}}\left(\xi_{1} \xi_{2} \eta_{1} \eta_{2}\right)^{|m| / 2} \exp \left(\frac{\left(\xi_{1}+\xi_{2}+\eta_{1}+\eta_{2}\right)}{2 a \nu}\right) \\
& \times \sum_{i, j} \frac{i ! j !(i+j+|m|+1-\nu)^{-1}}{(i+|m|) !(j+|m|) !} L_{i}^{|m|}\left(\frac{\xi_{1}}{a \nu}\right) \\
& \times L_{j}^{|m|}\left(\frac{\eta_{1}}{a \nu}\right) L_{i}^{|m|}\left(\frac{\xi_{2}}{a \nu}\right) L_{j}^{|m|}\left(\frac{\eta_{2}}{a \nu}\right) .
\end{aligned}
$$


Various different techniques have been utilized to arrive at these different forms for the SCGF in the literature. We have demonstrated in the above that they can all be derived from the basic Sturmian expansion by the straightforward application of well-known properties of special functions.

\section{The Dirac-Coulomb Green function}

The presentation of a functional form for the Dirac-Coulomb Green function is complicated by the presence of $4 \times 4$ matrix operators compounded with the absence of a relativistic equivalent of the Runge-Lenz vector. Nonetheless, as with our treatment of the SCGF, we are able to present a solution here based on the analysis of the defining differential equation. The DCGF $G\left(r_{1}, r_{2} ; E\right)$ corresponding to the energy variable $E$ is the $4 \times 4$ matrix function satisfying

$$
\begin{gathered}
{\left[\left(\boldsymbol{\alpha} \cdot \hat{\boldsymbol{r}}_{1}\right)\left(-\frac{\mathrm{i} \hbar c}{r_{1}} \frac{\mathrm{d}}{\mathrm{d} r_{1}} r_{1}+\frac{\mathrm{i} c}{r_{1}} \beta K\right)+\beta m c^{2}-\frac{\alpha Z \hbar c}{r_{1}}-E\right] G\left(\boldsymbol{r}_{1}, \boldsymbol{r}_{2} ; E\right)} \\
=\delta\left(\boldsymbol{r}_{1}-\boldsymbol{r}_{2}\right) I_{4}
\end{gathered}
$$

subject to suitable boundary conditions. Here, $K=\beta(\Sigma \cdot L+\hbar)$. Taking our lead from the treatment we made of the SCGF, we expand $G$ in the spinorial equivalent of spherical harmonics; that is, in terms of the spinor harmonics given by

$$
\chi_{\kappa}^{M}(\theta, \varphi)=\left(\begin{array}{c}
\frac{-\kappa}{|\kappa|}\left(\frac{\kappa+1 / 2-M}{2 \kappa+1}\right)^{1 / 2} Y_{\left|\kappa+\frac{1}{2}\right|-\frac{1}{2}, M-\frac{1}{2}}(\theta, \varphi) \\
\left(\frac{\kappa+1 / 2+M}{2 \kappa+1}\right)^{1 / 2} Y_{\left|\kappa+\frac{1}{2}\right|-\frac{1}{2}, M+\frac{1}{2}(\theta, \varphi)}
\end{array}\right) .
$$

Thus we try a solution of the form [21]

$$
G\left(\boldsymbol{r}_{1}, \boldsymbol{r}_{2} ; E\right)=\sum_{\kappa \mu}\left(\begin{array}{cc}
g_{\kappa}^{11}\left(r_{1}, r_{2}\right) \chi_{\kappa}^{\mu}\left(\hat{r}_{1}\right) \chi_{\kappa}^{\mu \dagger}\left(\hat{r}_{2}\right) & -\mathrm{i} g_{\kappa}^{12}\left(r_{1}, r_{2}\right) \chi_{\kappa}^{\mu}\left(\hat{r}_{1}\right) \chi_{-\kappa}^{\mu \dagger}\left(\hat{r}_{2}\right) \\
\mathrm{i} g_{\kappa}^{21}\left(r_{1}, r_{2}\right) \chi_{-\kappa}^{\mu}\left(\hat{r}_{1}\right) \chi_{\kappa}^{\mu \dagger}\left(\hat{r}_{2}\right) & g_{\kappa}^{22}\left(r_{1}, r_{2}\right) \chi_{-\kappa}^{\mu}\left(\hat{r}_{1}\right) \chi_{-\kappa}^{\mu \dagger}\left(\hat{r}_{2}\right)
\end{array}\right) .
$$

Noting thât

$$
\begin{aligned}
& \boldsymbol{\sigma} \cdot \hat{r} \chi_{\kappa}^{\mu}=-\chi_{-\kappa}^{\mu} \\
& (\boldsymbol{\sigma} \cdot \boldsymbol{L}+\hbar) \chi_{\kappa}^{\mu}=-\hbar \kappa \chi_{\kappa}^{\mu}
\end{aligned}
$$

and

$$
\delta\left(r_{1}-r_{2}\right) I_{4}=\delta\left(r_{1}-r_{2}\right) /\left(r_{1} r_{2}\right) \sum_{\kappa \mu}\left(\begin{array}{cc}
\chi_{\kappa}^{\mu}\left(\hat{r}_{1}\right) \chi_{\kappa}^{\mu \dagger}\left(\hat{r}_{2}\right) & 0 \\
0 & \chi_{-\kappa}^{\mu}\left(\hat{r}_{1}\right) \chi_{-\kappa}^{\mu \dagger}\left(\hat{r}_{2}\right)
\end{array}\right)
$$

and, employing the orthonormality properties of the spinor spherical harmonics we see that

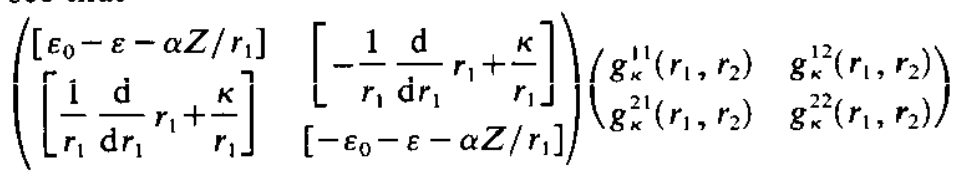

$$
\begin{aligned}
& =\frac{\delta\left(r_{1}-r_{2}\right)}{r_{1} r_{2}} I_{2} / \hbar c
\end{aligned}
$$


where

$$
\varepsilon_{0}=m c^{2} / \hbar c \quad \varepsilon=E / \hbar c .
$$

These are the radial equations we must solve. The trick is to perform a linear transformation which will reduce (3.7) to a form which will allow us to use the results of appendix 1. In [1] we found a transformation of the radial Dirac-Coulomb wavefunctions such that the transformed radial functions satisfied two uncoupled second-order differential equations. These equations were simple generalizations of the equation satisfied by the non-relativistic radial wavefunction. In the following we will modify that transformation to allow for much the same treatment of the Green functions.

Dispensing with the subscript ' $\kappa$ ' on the $g$ 's, it being understood that $\kappa$ is now a fixed parameter, we let

$$
\left(\begin{array}{ll}
f^{11}\left(r_{1}, r_{2}\right) & f^{12}\left(r_{1}, r_{2}\right) \\
f^{21}\left(r_{1}, r_{2}\right) & f^{22}\left(r_{1}, r_{2}\right)
\end{array}\right)=\underline{X}\left(\begin{array}{ll}
g^{11}\left(r_{1}, r_{2}\right) & g^{12}\left(r_{1}, r_{2}\right) \\
g^{21}\left(r_{1}, r_{2}\right) & g^{22}\left(r_{1}, r_{2}\right)
\end{array}\right) .
$$

where

$$
\underline{X}=\left(\begin{array}{cc}
1 & X \\
X & 1
\end{array}\right)
$$

and $X$ is to be determined. Then, since

$$
\underline{X}^{-1}=\left(1-X^{2}\right)^{-1}\left(\begin{array}{cc}
1 & -X \\
-X & 1
\end{array}\right)
$$

the radial equations $(3.7)$ become

$$
\begin{aligned}
& \left(\begin{array}{lll}
{\left[\varepsilon_{0}-\varepsilon-\frac{(\alpha Z+X \kappa-X)}{r_{1}}+X \frac{\mathrm{d}}{\mathrm{d} r_{1}}\right]} & {\left[-X \varepsilon_{0}+X \varepsilon+\frac{(\alpha Z X+\kappa-1)}{r_{1}}-\frac{\mathrm{d}}{\mathrm{d} r_{1}}\right]} \\
{\left[X \varepsilon_{0}+X \varepsilon+\frac{(\alpha Z X+\kappa+1)}{r_{1}}+\frac{\mathrm{d}}{\mathrm{d} r_{1}}\right]} & {\left[-\varepsilon_{0}-\varepsilon-\frac{(\alpha Z+X \kappa+X)}{r_{1}}-X \frac{\mathrm{d}}{\mathrm{d} r_{1}}\right]}
\end{array}\right) \\
& \times\left(\begin{array}{ll}
f^{11}\left(r_{1}, r_{2}\right) & f^{12}\left(r_{1}, r_{2}\right) \\
f^{21}\left(r_{1}, r_{2}\right) & f^{22}\left(r_{1}, r_{2}\right)
\end{array}\right)=\frac{\left(1-X^{2}\right)}{\hbar c} \frac{\delta\left(r_{1}-r_{2}\right)}{r_{1} r_{2}} I_{2} .
\end{aligned}
$$

We continue to follow the method employed in the reduction of the radial equations for the Dirac-Coulomb problem (see [1]) by eliminating the derivatives from the diagonal terms. This is achieved by premultiplying equation (3.12) by $\underline{X}^{-1}$ so that

$$
\begin{aligned}
& \left(\begin{array}{cc}
{\left[\left(1-X^{2}\right) \varepsilon_{0}-\left(1+X^{2}\right) \varepsilon-\frac{A}{r_{1}}\right]} & {\left[2 X \varepsilon+\frac{B}{r_{1}}-\left(1-X^{2}\right) \frac{d}{d r_{1}}\right]} \\
{\left[2 X \varepsilon+\frac{B}{r_{1}}+\left(1-X^{2}\right) \frac{d}{d r_{1}}\right]} & {\left[\left(X^{2}-1\right) \varepsilon_{0}-\left(1+X^{2}\right) \varepsilon-\frac{A}{r_{1}}\right]}
\end{array}\right) \\
& \times\left(\begin{array}{l}
f^{\prime \prime}\left(r_{1}, r_{2}\right) f^{12}\left(r_{1}, r_{2}\right) \\
f^{21}\left(r_{1}, r_{2}\right) f^{22}\left(r_{1}, r_{2}\right)
\end{array}\right)=\frac{\left(1-X^{2}\right)^{2}}{\hbar c} \frac{\delta\left(r_{1}-r_{2}\right)}{r_{1} r_{2}} \underline{X}^{-1}
\end{aligned}
$$

where

$$
A=\left(1+X^{2}\right) \alpha Z+2 X \kappa \quad B=2 \alpha Z X+\left(1+X^{2}\right) \kappa-\left(1-X^{2}\right) .
$$


We now choose $X$ in such a way that $A$ vanishes; that is, we let $X=(-\kappa+\gamma) / \alpha Z$ with $\gamma=\sqrt{\kappa^{2}-\alpha^{2} Z^{2}}$. The radial equations simplify considerably to

$$
\begin{gathered}
\left(\begin{array}{c}
{\left[\varepsilon_{0}-\varepsilon \kappa / \gamma\right]} \\
{\left[\frac{-\alpha Z \varepsilon}{\gamma}+\frac{\gamma+1}{r}+\frac{\mathrm{d}}{\mathrm{d} r}\right]}
\end{array} \begin{array}{c}
\left.\frac{-\alpha Z \varepsilon}{\gamma}+\frac{\gamma-1}{r}-\frac{\mathrm{d}}{\mathrm{d} r}\right] \\
{\left[-\varepsilon_{0}-\kappa \varepsilon / \gamma\right]}
\end{array}\right)\left(\begin{array}{ll}
f^{\prime 1}\left(r_{1}, r_{2}\right) & f^{12}\left(r_{1}, r_{2}\right) \\
f^{21}\left(r_{1}, r_{2}\right) & f^{22}\left(r_{1}, r_{2}\right)
\end{array}\right) \\
=\left(1-X^{2}\right) / \hbar c \frac{\delta\left(r_{1}-r_{2}\right)}{r_{1} r_{2}} \underline{X}^{-1} .
\end{gathered}
$$

The final step is to diagonalize the right-hand side of (3.15). Thus we define

$$
\left(\begin{array}{ll}
h^{11}\left(r_{1}, r_{2}\right) & h^{12}\left(r_{1}, r_{2}\right) \\
h^{21}\left(r_{1}, r_{2}\right) & h^{22}\left(r_{1}, r_{2}\right)
\end{array}\right)=\left(\begin{array}{ll}
f^{11}\left(r_{1}, r_{2}\right) & f^{12}\left(r_{1}, r_{2}\right) \\
f^{21}\left(r_{1}, r_{2}\right) & f^{22}\left(r_{1}, r_{2}\right)
\end{array}\right) \times \underline{X}
$$

so that, on post multiplying (3.15) by $X$, we have

$$
\begin{gathered}
\left(\left[\frac{-\alpha Z \varepsilon}{\gamma}+\frac{\gamma+1}{r_{1}}+\frac{\mathrm{d}}{\mathrm{d} r_{1}}\right] \begin{array}{c}
\left.\frac{-\alpha Z \varepsilon}{\gamma}+\frac{\gamma-1}{r_{1}}-\frac{\mathrm{d}}{\mathrm{d} r_{1}}\right] \\
{\left[-\varepsilon_{0}-\varepsilon \kappa / \gamma\right]}
\end{array}\right)\left(\begin{array}{ll}
h^{11}\left(r_{1}, r_{2}\right) & h^{12}\left(r_{1}, r_{2}\right) \\
h^{21}\left(r_{1}, r_{2}\right) & h^{22}\left(r_{1}, r_{2}\right)
\end{array}\right) \\
=\left(1-X^{2}\right) / \hbar c \frac{\delta\left(r_{1}-r_{2}\right)}{r_{1} r_{2}} I_{2} .
\end{gathered}
$$

The overall transformation is given by

$$
\left(\begin{array}{ll}
h^{11}\left(r_{t}, r_{2}\right) & h^{12}\left(r_{1}, r_{2}\right) \\
h^{21}\left(r_{1}, r_{2}\right) & h^{22}\left(r_{1}, r_{2}\right)
\end{array}\right)=\underline{X}\left(\begin{array}{ll}
g^{11}\left(r_{t}, r_{2}\right) & g^{12}\left(r_{1}, r_{2}\right) \\
g^{21}\left(r_{1}, r_{2}\right) & g^{22}\left(r_{1}, r_{2}\right)
\end{array}\right) X .
$$

We can now consider the four equations embodied in (3.17) in two pairs, since there is no mixing of the columns of the $h$-matrix. First,

$$
\begin{aligned}
& {\left[\varepsilon_{0}-\varepsilon \kappa / \gamma\right] h^{11}\left(r_{1}, r_{2}\right)+\left[-\frac{\alpha Z \varepsilon}{\gamma}+\frac{\gamma-1}{r_{1}}-\frac{\mathrm{d}}{\mathrm{d} r_{1}}\right] h^{21}\left(r_{1}, r_{2}\right)=\frac{\left(1-X^{2}\right)}{\hbar c} \frac{\delta\left(r_{1}-r_{2}\right)}{r_{1} r_{2}}} \\
& {\left[-\frac{\alpha Z \varepsilon}{\gamma}+\frac{\gamma+1}{r_{1}}+\frac{d}{d r_{1}}\right] h^{11}\left(r_{1}, r_{2}\right)-\left[\varepsilon_{0}+\varepsilon \kappa / \gamma\right] h^{21}\left(r_{1}, r_{2}\right)=0 .}
\end{aligned}
$$

Substituting (3.19b) into (3.19a) gives

$$
\begin{gathered}
{\left[\frac{\mathrm{d}^{2}}{\mathrm{~d} r_{1}^{2}}+\frac{2}{r_{1}} \frac{\mathrm{d}}{\mathrm{d} r_{1}}-\frac{\gamma(\gamma+1)}{r_{1}^{2}}+\frac{2 \alpha Z \varepsilon}{r_{1}}+\varepsilon^{2}-\varepsilon_{0}^{2}\right] h^{11}\left(r_{1}, r_{2}\right)} \\
=\left(\varepsilon_{0}+\varepsilon \kappa / \gamma\right) \frac{\left(X^{2}-1\right)}{\hbar c} \frac{\delta\left(r_{1}-r_{2}\right)}{r_{1} r_{2}}
\end{gathered}
$$

and

$$
h^{21}\left(r_{1}, r_{2}\right)=\gamma /\left(\varepsilon \kappa+\varepsilon_{0} \gamma\right)\left[-\frac{\alpha Z \varepsilon}{\gamma}+\frac{\gamma+1}{r_{1}}+\frac{\mathrm{d}}{\mathrm{d} r_{1}}\right] h^{\prime \prime}\left(r_{1}, r_{2}\right) .
$$

The second pair of equations is

$$
\begin{aligned}
& {\left[\varepsilon_{0}-\varepsilon \kappa / \gamma\right] h^{12}\left(r_{1}, r_{2}\right)+\left[-\frac{\alpha Z \varepsilon}{\gamma}+\frac{\gamma-1}{r_{1}}-\frac{\mathrm{d}}{\mathrm{d} r_{1}}\right] h^{22}\left(r_{1}, r_{2}\right)=0} \\
& {\left[-\frac{\alpha Z \varepsilon}{\gamma}+\frac{\gamma+1}{r_{1}}+\frac{\mathrm{d}}{\mathrm{d} r_{1}}\right] h^{12}\left(r_{1}, r_{2}\right)-\left[\varepsilon_{0}+\varepsilon \kappa / \gamma\right] h^{22}\left(r_{1}, r_{2}\right)=\frac{\left(1-X^{2}\right)}{\hbar c} \frac{\delta\left(r_{1}-r_{2}\right)}{r_{1} r_{2}} .}
\end{aligned}
$$


On substitution,

$$
\begin{gathered}
{\left[\frac{\mathrm{d}^{2}}{\mathrm{~d} r_{1}^{2}}+\frac{2}{r_{1}} \frac{\mathrm{d}}{\mathrm{d} r_{1}}-\frac{\gamma(\gamma-1)}{r_{1}^{2}}+\frac{2 \alpha Z \varepsilon}{r_{1}}+\varepsilon^{2}-\varepsilon_{0}^{2}\right] h^{22}\left(r_{1}, r_{2}\right)} \\
=\left(\varepsilon_{0}-\varepsilon \kappa / \gamma\right) \frac{\left(1-X^{2}\right)}{\hbar c} \frac{\delta\left(r_{1}-r_{2}\right)}{r_{1} r_{2}}
\end{gathered}
$$

and

$$
h^{12}\left(r_{1}, r_{2}\right)=\gamma /\left(\varepsilon \kappa-\varepsilon_{0} \gamma\right)\left[-\frac{\alpha Z \varepsilon}{\gamma}+\frac{\gamma-1}{r_{1}}-\frac{\mathrm{d}}{\mathrm{d} r_{1}}\right] h^{22}\left(r_{1}, r_{2}\right) .
$$

It is clear that the defining equations (3.20a) and (3.22a) of $h^{11}$ and $h^{22}$ are specific cases of the general equation examined in appendix 1 . We can thus solve them immediately.

Notice that the diagonal terms are clearly closely related. From appendix 1 we see that

$$
h^{11}\left(r_{1}, r_{2}\right)=\left(1-X^{2}\right)\left(\varepsilon \kappa+\varepsilon_{0} \gamma\right) /(\hbar c \gamma) g_{v \gamma}\left(r_{1}, r_{2} ; \omega\right)
$$

and

$$
h^{22}\left(r_{1}, r_{2}\right)=\left(1-X^{2}\right)\left(\varepsilon \kappa-\varepsilon_{0} \gamma\right) /(\hbar c \gamma) g_{v \gamma-1}\left(r_{1}, r_{2} ; \omega\right)
$$

where

$$
\begin{aligned}
& g_{\nu \lambda}\left(r_{1}, r_{2} ; \omega\right)=(2 \omega)^{2 \lambda+1}\left(r_{1} r_{2}\right)^{\lambda} \mathrm{e}^{-\left(r_{1}+r_{2}\right) \omega} \\
& \times \sum_{k=0}^{\infty} \frac{k !(k+1+\lambda-\nu)^{-1}}{\Gamma(2 \lambda+2+k)} L_{k}^{2 \lambda+1}\left(2 r_{1} \omega\right) L_{k}^{2 \lambda+1}\left(2 r_{2} \omega\right) \\
& \omega=\sqrt{\varepsilon_{0}^{2}-\varepsilon^{2}} \text { and } \nu=\alpha Z \varepsilon / \sqrt{\varepsilon_{0}^{2}-\varepsilon^{2}} .
\end{aligned}
$$

We may derive various other representations just as we did for the scGF. Hence, in exactly the same manner as was used in section 2 , we find $g_{\nu \lambda}\left(r_{1}, r_{2} ; \omega\right)$

$$
\begin{aligned}
= & 1 / \sqrt{r_{1} r_{2}} \mathrm{e}^{\left(r_{1}+r_{2}\right) \omega} \int_{0}^{\infty} \int_{0}^{\infty} \mathrm{e}^{-s_{1}-s_{2} J_{2 \lambda+1}\left(2 \sqrt{\left.2 \omega r_{1} s_{1}\right)}\right.} \\
& \times J_{2 \lambda+1}\left(2 \sqrt{2 \omega r_{2} s_{2}}\right) \sum_{k=0}^{\infty} \frac{\left(s_{1} s_{2}\right)^{k+\lambda+\frac{1}{2}}}{\Gamma(2 \lambda+2+k) k !(\lambda+1+k-\nu)} \mathrm{d} s_{1} \mathrm{~d} s_{2} \\
= & 1 / \sqrt{r_{1} r_{2}} \int_{0}^{1} t^{-\nu-\frac{1}{2}}(1-t) \exp \left(-\omega\left[r_{1}+r_{2}\right][1+t] /[1-t]\right) \\
& \times I_{2 \lambda+1}\left(4 \omega \sqrt{r_{1} r_{2} t} /[1-t]\right) \mathrm{d} t \\
= & 1 / \sqrt{r_{1} r_{2}} \mathrm{e}^{\left(r_{1}+r_{2}\right) \omega} \int_{0}^{1}(1-s)^{-\nu-\frac{1}{2} s^{-1}} \exp \left(-2 \omega\left[r_{1}+r_{2}\right] / s\right) \\
& \times I_{2 \lambda+1}\left(4 \omega \sqrt{\left.r_{1} r_{2}[1-s] / s\right) \mathrm{d} s}\right. \\
= & 1 / \sqrt{r_{1} r_{2}} \int_{0}^{\infty}\left(\operatorname{coth} \frac{\sigma}{2}\right)^{2 \nu} \exp \left(-\omega\left[r_{1}+r_{2}\right] \cosh \sigma\right) \\
& \times I_{2 \lambda+1}\left(2 \omega \sqrt{r_{1} r_{2}} \sinh \sigma\right) \mathrm{d} \sigma \\
= & (1 / 2 \omega) \frac{\Gamma(\lambda+1-\nu)}{\Gamma(2 \lambda+2)} \frac{1}{r_{1} r_{2}} M_{\nu, \lambda+\frac{!}{2}\left(2 \omega r_{<}\right) W_{u, \lambda+\frac{1}{2}}\left(2 \omega r_{>}\right)}
\end{aligned}
$$


The off-diagonal terms can be simply calculated using (3.20b) and $(3.22 b)$. The Sturmian expansion is of interest since it is not quite what one might expect. Using relations (A2.2) and (A2.4) derived in appendix 2, we see that

$$
\begin{aligned}
h^{21}\left(r_{1}, r_{2}\right)= & h^{12}\left(r_{2}, r_{1}\right) \\
= & \left(1-X^{2}\right) /(2 \hbar c \gamma)\left(\delta\left(r_{1}-r_{2}\right) / \sqrt{r_{1} r_{2}}+(2 \omega)^{2 \gamma+1} r_{1}^{\gamma-1} r_{2}^{\gamma}\right. \\
& \left.\times \mathrm{e}^{-\omega\left(r_{1}+r_{2}\right)} \sum_{k=0}^{\infty} \frac{(k+1) !(\gamma+k+1-\nu)^{-1}}{\Gamma(2 \gamma+1+k)} L_{k+1}^{2 \gamma-1}\left(2 \omega r_{1}\right) L_{k}^{2 \gamma+1}\left(2 \omega r_{2}\right)\right) .
\end{aligned}
$$

We note first that $h^{i j}\left(r_{1}, r_{2}\right)=h^{j i}\left(r_{2}, r_{1}\right)$. Thus, in spite of the seemingly asymmetrical defining equations for the radial DCGFs, they are nonetheless symmetrical in $r_{1}$ and $r_{2}$. Next, we notice that the off-diagonal terms are not simple Sturmian expansions. The delta function appearing in (3.31) is required for the correct calculation of the matrix elements.

A Sturmian form of the radial Dirac-Coulomb Green function was obtained previously by solving the second-order problem [14]. The method is quite complicated and the solution presented, while apparently equivalent to the one above, does not explicitly display the delta functions.

We can find expressions for $h^{12}$ and $h^{21}$ in terms of Whittaker functions also. Using the relations given in appendix 4 for the derivatives of $M$ and $W$, and the defining equations $(3.20 b)$ and $(3.22 b)$, we find that

$$
\begin{aligned}
h^{21}\left(r_{1}, r_{2}\right)= & h^{12}\left(r_{2}, r_{1}\right) \\
= & \frac{\left(1-X^{2}\right) \Gamma(\gamma+1-\nu)}{2 \gamma \hbar c \Gamma(2 \gamma+2)} \frac{1}{r_{1} r_{2}}\left[2 \gamma(2 \gamma+1) \theta\left(r_{2}-r_{1}\right) M_{\nu, \gamma-\frac{1}{2}}\left(2 \omega r_{1}\right) W_{\nu, \gamma+\frac{1}{2}}\left(2 \omega r_{2}\right)\right. \\
& \left.-(\nu+\gamma) \theta\left(r_{1}-r_{2}\right) W_{\nu, \gamma-\frac{1}{2}}\left(2 \omega r_{1}\right) M_{\nu, \gamma+\frac{1}{2}}\left(2 \omega r_{2}\right)\right]
\end{aligned}
$$

where $\theta(r)$ is the unit Heaviside function. Notice that no delta function appears in this expression. Notice also the lack of symmetry in the coefficients multiplying the Whittaker functions.

To complete the analysis, we present a brief examination of the non-relativistic limit of the DCGF we have just calculated. It will be useful to express the original radial functions in terms of the radial functions we have obtained. Thus, inverting (3.18) we find (omitting the variables for a moment)

$$
\begin{aligned}
\left(\begin{array}{ll}
g^{11} & g^{12} \\
g^{21} & g^{22}
\end{array}\right)= & \frac{1}{\left(1-X^{2}\right)^{2}} \\
& \times\left(\begin{array}{cc}
h^{11}-X\left(h^{12}+h^{21}\right)+X^{2} h^{22} & -X\left(h^{11}+h^{22}\right)+h^{12}+X^{2} h^{21} \\
-X\left(h^{11}+h^{22}\right)+X^{2} h^{12}+h^{21} & X^{2} h^{11}-X\left(h^{12}+h^{21}\right)+h^{22}
\end{array}\right) .
\end{aligned}
$$

As in the reduction of the Dirac-Coulomb wavefunctions to their non-relativistic limits (see [1]), two separate situations obtain, depending on the sign of $\kappa$. We use the Sturmian expansion throughout.

When $\kappa=|\kappa|$, we set $\kappa=l, \omega \simeq 1 / a \nu$ and note

$$
\begin{array}{ll}
X \simeq-\alpha Z / 2 l \quad & 1-X^{2} \simeq 1 \quad \varepsilon \kappa+\varepsilon_{0} \gamma \simeq 2 l / a \alpha Z \\
\varepsilon \kappa-\varepsilon_{0} \gamma \simeq \alpha Z\left(\nu^{2}-l^{2}\right) / 2 a l \nu^{2} &
\end{array}
$$


and $\gamma \simeq l$. Then the lowest-order term in $\alpha Z$ is $g^{11}$ and is the non-relativistic limit of $h^{11}$, which is itself easily seen to equal $g_{l}(\nu)$. Hence, for $\kappa=|\kappa|=l$,

$$
\left(\begin{array}{ll}
g^{11} & g^{12} \\
g^{21} & g^{22}
\end{array}\right) \simeq\left(\begin{array}{ll}
g & 0 \\
0 & 0
\end{array}\right)
$$

When $\kappa=-|\kappa|$, we set $\kappa=-I-1, \omega \simeq 1 / a \nu$, and note

$$
X=2(l+1) / \alpha Z \quad 1-X^{2} \simeq-4(l+1)^{2} / \alpha^{2} Z^{2}
$$

$$
\varepsilon \kappa+\varepsilon_{0} \gamma \simeq-\alpha Z(\nu-l-1)(\nu+l+1) /\left[2 a(l+1) \nu^{2}\right] \quad \varepsilon \kappa-\varepsilon_{0} \gamma \simeq-2(l+1) /(a \alpha Z)
$$

and $y \simeq l+1$. The lowest-order term in $\alpha Z$ is again $g^{11}$ but is now the non-relativistic limit of $X^{2} h^{22} /\left(1-X^{2}\right)^{2}$, which again is equal to $g_{l}(\nu)$. Hence, for $\kappa=-|\kappa|=-1-1$, we arrive at equation (3.34) again.

Thus, in the non-relativistic limit, all terms in the $2 \times 2$ matrix of radial DCGFs but the leading diagonal term vanish, and the non-vanishing element is just the radial SCGF as we might have expected.

\section{Recursion relations for the radial Green functions}

In appendix 2 we show that

$$
\begin{aligned}
{\left[\lambda\left(\frac{\mathrm{d}}{\mathrm{d} r_{1}}+\frac{\lambda+1}{r_{1}}\right)-\omega \nu\right] g_{\nu \lambda}\left(r_{1}, r_{2} ; \omega\right) } \\
=\frac{1}{2}\left\{\delta\left(r_{1}-r_{2}\right) / \sqrt{r_{1} r_{2}}+(2 \omega)^{2 \lambda+1} r_{1}^{\lambda-1} r_{2}^{\lambda} \mathrm{e}^{-\left(r_{1}+r_{2}\right) \omega}\right. \\
\left.\quad \times \sum_{k=0}^{\infty} \frac{(k+1) !}{\Gamma(2 \lambda+1+k)(\lambda+1+k-\nu)} L_{k+1}^{2 \lambda-1}\left(2 \omega r_{1}\right) L_{k}^{2 \lambda+1}\left(2 \omega r_{2}\right)\right\}
\end{aligned}
$$

and since $g_{\nu \lambda}$ is symmetrical in $r_{1}, r_{2}$ and the Dirac delta function is even,

$$
\begin{aligned}
{\left[\lambda\left(\frac{\mathrm{d}}{\mathrm{d} r_{2}}-\frac{\lambda-1}{r_{2}}\right)+\omega \nu\right] g_{\nu \lambda-1}\left(r_{1}, r_{2} ; \omega\right) } \\
=-\frac{1}{2}\left\{\delta\left(r_{1}-r_{2}\right) / \sqrt{r_{1} r_{2}}+(2 \omega)^{2 \lambda+1} r_{1}^{\lambda-1} r_{2}^{\lambda} \mathrm{e}^{-\left(r_{3}+r_{2}\right) \omega}\right. \\
\left.\quad \times \sum_{k=0}^{\infty} \frac{(k+1) !}{\Gamma(2 \lambda+1+k)(\lambda+1+k-\nu)} L_{k+1}^{2 \lambda-1}\left(2 \omega r_{1}\right) L_{k}^{2 \lambda+1}\left(2 \omega r_{2}\right)\right\} .
\end{aligned}
$$

Thus

$$
\left[\lambda\left(\frac{\mathrm{d}}{\mathrm{d} r_{1}}+\frac{\lambda+1}{r_{1}}\right)-\omega \nu\right] g_{\nu \lambda}\left(r_{1}, r_{2} ; \omega\right)=-\left[\lambda\left(\frac{\mathrm{d}}{\mathrm{d} r_{2}}-\frac{\lambda-1}{r_{2}}\right)+\omega \nu\right] g_{\nu \lambda \lambda-1}\left(r_{1}, r_{2} ; \omega\right) .
$$

This fundamental recursion relation, together with the defining differential equation 
for $g_{\nu \lambda}$, allows us easily to derive both $\lambda$ raising and lowering operators for $g_{\nu \lambda}$. Thus

$$
\begin{gathered}
{\left[\lambda\left(\frac{\mathrm{d}}{\mathrm{d} r_{1}}+\frac{\lambda+1}{r_{1}}\right)-\omega \nu\right]\left[\lambda\left(\frac{\mathrm{d}}{\mathrm{d} r_{2}}+\frac{\lambda+1}{r_{2}}\right)-\omega \nu\right] g_{\nu \lambda}\left(r_{1}, r_{2} ; \omega\right)} \\
=-\left[\lambda\left(\frac{\mathrm{d}}{\mathrm{d} r_{2}}+\frac{\lambda+1}{r_{2}}\right)-\omega \nu\right]\left[\lambda\left(\frac{\mathrm{d}}{\mathrm{d} r_{2}}-\frac{\lambda-1}{r_{2}}\right)+\omega \nu\right] g_{\nu \lambda-1}\left(r_{1}, r_{2} ; \omega\right) \\
=\left[\lambda^{2}\left(\frac{\mathrm{d}^{2}}{\mathrm{~d} r_{2}^{2}}+\frac{2}{r_{2}} \frac{\mathrm{d}}{\mathrm{d} r_{2}}-\frac{\lambda(\lambda-1)}{r_{2}^{2}}+\frac{2 \omega \nu}{r_{2}}\right)-\omega^{2} \nu^{2}\right] g_{\nu \lambda-1}\left(r_{1}, r_{2} ; \omega\right)
\end{gathered}
$$

and, given the defining equation of $g_{\nu \lambda-1}$, we have the following $\lambda$ lowering relation for $g_{\nu \mathrm{A}}$ :

$$
\begin{array}{r}
{\left[\lambda\left(\frac{\mathrm{d}}{\mathrm{d} r_{1}}+\frac{\lambda+1}{r_{1}}\right)-\omega \nu\right]\left[\lambda\left(\frac{\mathrm{d}}{\mathrm{d} r_{2}}+\frac{\lambda+1}{r_{2}}\right)-\omega \nu\right] g_{\nu \lambda}\left(r_{1}, r_{2} ; \omega\right)} \\
=\lambda^{2} \delta\left(r_{1}-r_{2}\right) /\left(r_{1} r_{2}\right)+\omega^{2}\left(\nu^{2}-\lambda^{2}\right) g_{\nu \lambda-1}\left(r_{1}, r_{2} ; \omega\right) .
\end{array}
$$

In just the same way we can deduce a $\lambda$ raising relation:

$$
\begin{gathered}
{\left[\lambda\left(\frac{\mathrm{d}}{\mathrm{d} r_{1}}-\frac{\lambda-1}{r_{1}}\right)+\omega \nu\right]\left[\lambda\left(\frac{\mathrm{d}}{\mathrm{d} r_{2}}-\frac{\lambda-1}{r_{2}}\right)+\omega \nu\right] g_{\nu \lambda-1}\left(r_{1}, r_{2} ; \omega\right)} \\
=-\left[\lambda\left(\frac{\mathrm{d}}{\mathrm{d} r_{1}}-\frac{\lambda-1}{r_{1}}\right)+\omega \nu\right]\left[\lambda\left(\frac{\mathrm{d}}{\mathrm{d} r_{1}}+\frac{\lambda+1}{r_{1}}\right)-\omega \nu\right] g_{\nu \lambda}\left(r_{1}, r_{2} ; \omega\right) \\
=\lambda^{2} \delta\left(r_{1}-r_{2}\right) /\left(r_{1} r_{2}\right)+\omega^{2}\left(\nu^{2}-\lambda^{2}\right) g_{\nu \lambda}\left(r_{1}, r_{2} ; \omega\right) .
\end{gathered}
$$

In appendix 3 we give an alternate derivation of these relations using double Laplace transforms. The advantage of that approach is that no specific properties of Laguerre polynomials need to assumed.

These relations bear a striking resemblance to those representing $\lambda$ raising and lowering operators of the wavefunctions [1]. Apart from the obvious fact that we are now dealing with two variables, the main difference is the presence of the $\delta$-function in (4.4) and (4.5).

It is worthwhile to write down the explicit forms of the recursion relations as they apply to the SCGF and DCGF. In the non-relativistic case we see that

$$
\begin{aligned}
& {\left[l\left(\frac{\mathrm{d}}{\mathrm{d} r_{1}}+\frac{l+1}{r_{1}}\right)-1 / a\right] g_{l}\left(r_{1}, r_{2} ; \nu\right)} \\
& =m / \hbar^{2}\left\{\delta\left(r_{1}-r_{2}\right) / \sqrt{r_{1} r_{2}}+(2 / a \nu)^{2 l+1} r_{1}^{l-1} r_{2}^{\prime} \mathrm{e}^{-\left(r_{1}+r_{2}\right) / a \nu}\right. \\
& \left.\times \sum_{k=0}^{\infty} \frac{(k+1) !}{\Gamma(2 l+1+k)(l+1+k-\nu)} L_{k+1}^{2 l-1}\left(\frac{2 r_{1}}{a \nu}\right) L_{k}^{2 l+1}\left(\frac{2 r_{2}}{a \nu}\right)\right\} \\
& =\left[l\left(\frac{\mathrm{d}}{\mathrm{d} r_{2}}-\frac{l-1}{r_{2}}\right)+1 / a\right] g_{l-1}\left(r_{1}, r_{2} ; \nu\right) \\
& {\left[l\left(\frac{\mathrm{d}}{\mathrm{d} r_{1}}+\frac{l+1}{r_{1}}\right)-1 / a\right]\left[l\left(\frac{\mathrm{d}}{\mathrm{d} r_{2}}+\frac{l+1}{r_{2}}\right)-1 / a\right] g_{l}\left(r_{1}, r_{2} ; \nu\right)} \\
& =2 m / \hbar^{2} l^{2} \delta\left(r_{1}-r_{2}\right) / r_{1} r_{2}+\left(\nu^{2}-l^{2}\right) /(a \nu)^{2} g_{l-1}\left(r_{1}, r_{2} ; \nu\right)
\end{aligned}
$$


and

$$
\begin{gathered}
{\left[l\left(\frac{\mathrm{d}}{\mathrm{d} r_{1}}-\frac{l-1}{r_{1}}\right)+1 / a\right]\left[l\left(\frac{\mathrm{d}}{\mathrm{d} r_{2}}-\frac{l-1}{r_{2}}\right)+1 / a\right] g_{l-1}\left(r_{1}, r_{2} ; \nu\right)} \\
=2 m / \hbar^{2} l^{2} \delta\left(r_{1}-r_{2}\right) / r_{1} r_{2}+\left(\nu^{2}-l^{2}\right) /(a \nu)^{2} g_{l}\left(r_{1}, r_{2} ; \nu\right) .
\end{gathered}
$$

In the relativistic case we have already derived certain relations appertaining to the off-diagonal terms of the radial DCGF. The further relations we present now are

$$
\begin{aligned}
& \left(\varepsilon \kappa-\varepsilon_{0} \gamma\right)\left[\gamma\left(\frac{\mathrm{d}}{\mathrm{d} r_{1}}+\frac{\gamma+1}{r_{1}}\right)-\alpha Z \varepsilon\right] h^{11}\left(r_{1}, r_{2}\right) \\
& =-\left(\varepsilon \kappa+\varepsilon_{0} \gamma\right)\left[\gamma\left(\frac{\mathrm{d}}{\mathrm{d} r_{2}}-\frac{\gamma-1}{r_{2}}\right)+\alpha Z \varepsilon\right] h^{22}\left(r_{1}, r_{2}\right) \\
& {\left[\gamma\left(\frac{\mathrm{d}}{\mathrm{d} r_{1}}+\frac{\gamma+1}{r_{1}}\right)-\alpha Z \varepsilon\right]\left[\gamma\left(\frac{\mathrm{d}}{\mathrm{d} r_{2}}+\frac{\gamma+1}{r_{2}}\right)-\alpha Z \varepsilon\right] h^{11}\left(r_{1}, r_{2}\right)} \\
& =\left(1-X^{2}\right)\left(\varepsilon \kappa+\varepsilon_{0} \gamma\right) \gamma / \hbar c \delta\left(r_{1}-r_{2}\right) / r_{1} r_{2}+\left(\varepsilon \kappa+\varepsilon_{0} \gamma\right)^{2} h^{22}\left(r_{1}, r_{2}\right) \\
& {\left[\gamma\left(\frac{\mathrm{d}}{\mathrm{d} r_{1}}-\frac{\gamma-1}{r_{1}}\right)+\alpha Z \varepsilon\right]\left[\gamma\left(\frac{\mathrm{d}}{\mathrm{d} r_{2}}-\frac{\gamma-1}{r_{2}}\right)+\alpha Z \varepsilon\right] h^{22}\left(r_{1}, r_{2}\right)} \\
& =\left(1-X^{2}\right)\left(\varepsilon \kappa-\varepsilon_{0} \gamma\right) \gamma / \hbar c \delta\left(r_{1}-r_{2}\right) / r_{1} r_{2}+\left(\varepsilon \kappa-\varepsilon_{0} \gamma\right)^{2} h^{11}\left(r_{1}, r_{2}\right) .
\end{aligned}
$$

Here we have employed a shorthand notation, omitting the dependence on $\kappa$ and $\omega$ from $h$, and the dependence on $\kappa$ from $X$.

In the context of a discussion on the reduced SCGF, Johnson and Hirschfelder [22] have presented recursion relations of the radial SCGF. Their relations differ from our (4.7) and (4.8), in that they are written in terms of $r_{<}$and $r_{>}$and are therefore useful only when considering the radial scGF written in terms of Whittaker functions.

\section{Double Laplace transforms of the radial Coulomb Green function}

Quite general matrix elements of both the non-relativistic and relativistic Coulomb Green functions can be calculated once we know the double Laplace transforms (DLTs) of the radial functions, although in specific calculations better techniques may be available. We have presented various forms of the DLTs for the non-relativistic case in [19]. In this section we present general forms for the DLT applicable to both the SCGF and DCGF.

Some of the compact expressions presented in [19] for the DLT of $g_{t}$ will not be possible. However, there are several expressions which do still obtain, in somewhat modified form, and they are given below. The results will be presented without proof; the proofs consist of simple generalizations of those given previously. We need only really consider the DLTs of the diagonal elements of the DCGF, since the DLTs of the off-diagonal elements are simply related to them by equations $(3.20 b)$ and $(3.22 b)$.

Our general DLT is taken to be

$$
K_{\mu_{1} \mu_{2}}^{\nu \lambda}\left(p_{1}, p_{2} ; \omega\right)=\int_{0}^{\infty} \int_{0}^{\infty} r_{1}^{\mu_{1}} r_{2}^{\mu}=\mathrm{e}^{-\left(p_{1} r_{1}+p_{2} r_{2}\right) \omega} g_{\nu \lambda}\left(r_{1}, r_{2} ; \omega\right) \mathrm{d} r_{1} \mathrm{~d} r_{2}
$$

where the $\mu$ 's are not necessarily integers, and we have defined $g$ in appendix 1 . 
We begin with the integral representation of $g$, equation (3.28). Then the successive application of the properties of generalized hypergeometric functions shows that

$$
\begin{aligned}
& K_{\mu_{1} \mu_{2}}^{\nu \lambda}\left(p_{1}, p_{2} ; \omega\right) \\
& =(1 / 2 \omega)^{\mu_{1}+\mu_{2}+1} \Gamma\left(\mu_{1}+\mu_{2}+1\right) \\
& \times \sum_{k=0}^{\infty} \frac{\Gamma\left(\lambda+\mu_{1}+k+1\right) \Gamma\left(\lambda+\mu_{2}+k+1\right) \Gamma(\lambda+k+1-\nu)}{\Gamma(2 \lambda+2+k) k ! \Gamma\left(\lambda+\mu_{1}+\mu_{2}+k+2-\nu\right)} \\
& \times F_{1}\left(\mu_{1}+\mu_{2}+1 ; \lambda+\mu_{1}+k+1, \lambda+\mu_{2}+k+1\right. \text {; } \\
& \left.\lambda+\mu_{1}+\mu_{2}+k+2-\nu ;\left(\frac{1-p_{1}}{2}\right),\left(\frac{1-p_{2}}{2}\right)\right) \\
& =(1 / 2 \omega)^{\mu_{1}+\mu_{2}+1} \\
& \times \sum_{m_{1}, m_{2}}^{\infty} \frac{\Gamma\left(\mu_{1}+\mu_{2}+m_{1}+m_{2}+1\right) \Gamma\left(\lambda+\mu_{1}+m_{1}+1\right) \Gamma\left(\lambda+\mu_{2}+m_{2}+1\right) \Gamma(\lambda+1-\nu)}{\Gamma\left(\lambda+\mu_{1}+\mu_{2}+m_{1}+m_{2}+2-\nu\right) \Gamma(2 \lambda+2) m_{1} ! m_{2} !} \\
& \times\left(\frac{1-p_{1}}{2}\right)^{m_{1}}\left(\frac{1-p_{2}}{2}\right)^{m_{2}} \\
& \times_{3} F_{2}\left(\begin{array}{c}
\mu_{1}+m_{1}+\lambda+1, \mu_{2}+m_{2}+\lambda+1, \lambda+1-\nu \\
\lambda+\mu_{1}+\mu_{2}+m_{1}+m_{2}+2-\nu, 2 \lambda+2
\end{array} ; 1\right) \\
& =(1 / 2 \omega)^{\mu_{1}+\mu_{2}+1} \Gamma\left(\lambda+\mu_{1}+1\right) \Gamma\left(\lambda+\mu_{2}+1\right) / \Gamma(\lambda+1+\nu) \\
& \times \sum_{k=0}^{\infty} \frac{\Gamma\left(\mu_{1}+\mu_{2}+k+1\right) \Gamma(\lambda+1+\nu+k)}{\Gamma\left(\lambda+\mu_{1}+k+2\right) \Gamma\left(\lambda+\mu_{2}+k+2\right)} \\
& \times F_{2}\left(\mu_{1}+\mu_{2}+k+1 ; \lambda+\mu_{1}+1, \lambda+\mu_{2}+1\right. \text {; } \\
& \left.\lambda+\mu_{1}+k+2, \lambda+\mu_{2}+k+2 ;\left(\frac{1-p_{1}}{2}\right),\left(\frac{1-p_{2}}{2}\right)\right) .
\end{aligned}
$$

The generalized hypergeometric function ${ }_{3} F_{2}$ and the Appell functions $F_{1}$ and $F_{2}$ are defined in appendix 4.

The Sturmian expansion (3.25) is again useful for calculating the DLTs. In fact, the off-diagonal elements of the DCGF can also be treated in this form.

$$
\begin{aligned}
K_{\mu_{1} \mu_{2}}^{\nu \lambda}\left(p_{1}, p_{2} ; \omega\right) & \\
= & 2^{2 \lambda+1} \omega^{-\mu_{1}-\mu_{2}-1}\left(p_{1}+1\right)^{-\mu_{1}-\lambda-1}\left(p_{2}+1\right)^{-\mu_{2}-\lambda-1} \\
& \times \sum_{k=0}^{\infty} \frac{\Gamma\left(\mu_{1}+\lambda+k+1\right) \Gamma\left(\mu_{2}+\lambda+k+1\right)}{\Gamma(2 \lambda+2+k) k !(\lambda+1+k-\nu)}\left(\frac{p_{1}-1}{p_{1}+1}\right)^{k}\left(\frac{p_{2}-1}{p_{2}+1}\right)^{k} \\
& \times{ }_{2} F_{1}\left(-k, \lambda+1-\mu_{1} ;-\mu_{1}-\lambda-k ; \frac{p_{1}+1}{p_{1}-1}\right) \\
& \times{ }_{2} F_{1}\left(-k, \lambda+1-\mu_{2} ;-\mu_{2}-\lambda-k ; \frac{p_{2}+1}{p_{2}-1}\right)
\end{aligned}
$$


and

$$
\begin{aligned}
\bar{K}_{\mu_{1} \mu_{2}}^{\nu \gamma}\left(p_{1}, p_{2} ; \omega\right) & \int_{0}^{\infty} r_{1}^{\mu_{1}} r_{2}^{\mu_{2}} \mathrm{e}^{-\left(r_{1} p_{1}+r_{2} p_{2}\right) \omega} h^{21}\left(r_{1}, r_{2}\right) \mathrm{d} r_{1} \mathrm{~d} r_{2} \\
= & \left(1-X^{2}\right) /(2 \hbar c \gamma)(1 / \omega)^{\mu_{1}+\mu_{2}} \\
& \times\left[\left(p_{1}+p_{2}\right)^{-\mu_{1}-\mu_{2}} \Gamma\left(\mu_{1}+\mu_{2}\right)+2^{2 \gamma+1}\left(p_{1}+1\right)^{-\mu_{1}-\gamma}\left(p_{2}+1\right)^{-\mu_{2}-\gamma-1}\right. \\
& \times \sum_{k=0}^{\infty} \frac{\Gamma\left(\mu_{1}+\gamma+k+1\right) \Gamma\left(\mu_{2}+\gamma+k+1\right)}{\Gamma(2 \gamma+1+k)(\gamma+k+1-\nu) k !}\left(\frac{p_{1}-1}{p_{1}+1}\right)^{k+1}\left(\frac{p_{2}-1}{p_{2}+1}\right)^{k} \\
& \times{ }_{2} F_{1}\left(-k-1, \gamma-\mu_{1} ;-\mu_{1}-\gamma-k ; \frac{p_{1}+1}{p_{1}-1}\right) \\
& \left.\times{ }_{2} F_{1}\left(-k, \gamma-\mu_{2}+1 ;-\mu_{2}-\gamma-k ; \frac{p_{2}+1}{p_{2}-1}\right)\right] .
\end{aligned}
$$

The last two expressions are all that is needed to determine the relativistic matrix elements of the Dirac-Coulomb Green function.

Finally, there is a representation of $K$ based on the form of $g$ given by (3.26).

$$
\begin{aligned}
K_{\mu_{2} \mu_{2}}^{\nu \lambda}\left(p_{1}, p_{2} ; \omega\right) & \\
= & 2^{2 \lambda+1} \omega^{-\mu_{1}-\mu_{2}-1}\left(p_{1}+1\right)^{-\lambda-\mu_{1}-1}\left(p_{2}+1\right)^{-\lambda-\mu_{2}-1} \\
& \times \frac{\Gamma\left(\mu_{1}+\lambda+1\right) \Gamma\left(\mu_{2}+\lambda+1\right)}{[\Gamma(2 \lambda+2)]^{2}} \sum_{k=0}^{\infty} \frac{\Gamma(2 \lambda+2+k)}{k !(\lambda+1+k-\nu)} \\
& \times{ }_{2} F_{1}\left(\mu_{1}+\lambda+1,-k ; 2 \lambda+2 ; \frac{2}{p_{1}+1}\right) \\
& \times{ }_{2} F_{1}\left(\mu_{2}+\lambda+1,-k ; 2 \lambda+2 ; \frac{2}{p_{2}+1}\right) .
\end{aligned}
$$

\section{Matrix element recursion relations}

A further method of obtaining matrix elements of the radial CGF consists of a generalization of the hypervirial theorem, well known in connection with the calculation of matrix elements of the Coulomb wavefunctions. The method actually generates relations between matrix elements, so that in general a small number of explicit calculations will lead immediately to a whole series of matrix elements.

Suppose that $A_{\nu^{\prime} \lambda^{\prime} \omega^{\prime}}^{\nu \omega^{\prime}}$ and $B_{\nu^{\lambda} \lambda^{\prime} \omega^{\prime}}^{\nu \lambda \omega^{\prime}}$ are operators related in such a manner that

$$
\begin{gathered}
{\left[\frac{\mathrm{d}^{2}}{\mathrm{~d} r^{2}}+\frac{2}{r} \frac{\mathrm{d}}{\mathrm{d} r}\right.} \\
\left.-\frac{\lambda^{\prime}\left(\lambda^{\prime}+1\right)}{r^{2}}+\frac{2 \omega^{\prime} \nu^{\prime}}{r}-\omega^{\prime 2}\right] A_{\nu^{\prime} \lambda^{\prime} \omega^{\prime}}^{\nu \lambda \omega}\left(r^{\lambda} \mathrm{e}^{-\omega r} L_{\nu-\lambda-1}^{2 \lambda+1}(2 \omega r)\right) \\
=B_{\nu^{\prime} \lambda^{\prime} \omega}^{\nu \lambda \omega} \cdot\left(r^{\lambda} \mathrm{e}^{-\omega \omega r} L_{\nu-\lambda-1}^{2 \lambda+1}(2 \omega r)\right)
\end{gathered}
$$


where $\nu-\lambda-1$ is a non-negative integer. Then the hypervirial method is based on the fact that since

$\left[\frac{\mathrm{d}^{2}}{\mathrm{~d} r_{1}^{2}}+\frac{2}{r_{1}} \frac{\mathrm{d}}{\mathrm{d} r_{1}}-\frac{\lambda(\lambda+1)}{r_{1}^{2}}+\frac{2 \omega \nu}{r_{1}}-\omega^{2}\right] g_{\nu \lambda}\left(r_{1}, r_{2} ; \omega\right)=-\delta\left(r_{1}-r_{2}\right) /\left(r_{1} r_{2}\right)$

straightforward integration by parts shows that

$$
\begin{aligned}
\int_{0}^{\infty} r_{1}^{2} g_{\nu^{\prime} \lambda^{\prime}}\left(r_{1},\right. & \left.r_{2} ; \omega^{\prime}\right) B_{\nu^{\prime} \lambda^{\prime} \omega^{\prime}}^{\nu \lambda \omega}\left(r_{1}^{\lambda} e^{-\omega r_{1}} L_{\nu-\lambda-1}^{2 \lambda+1}\left(2 \omega r_{1}\right)\right) \mathrm{d} r_{1} \\
= & \int_{0}^{\infty} r_{1}^{2}\left[\frac{\mathrm{d}^{2}}{\mathrm{~d} r_{1}^{2}}+\frac{2}{r_{1}} \frac{\mathrm{d}}{\mathrm{d} r_{1}}-\frac{\lambda^{\prime}\left(\lambda^{\prime}+1\right)}{r_{1}^{2}}+\frac{2 \omega^{\prime} \nu^{\prime}}{r_{1}}-\omega^{\prime 2}\right] g_{\nu^{\prime} \lambda^{\prime}}\left(r_{1}, r_{2} ; \omega^{\prime}\right) \\
& \times A_{v^{\prime} \lambda^{\prime} \omega^{\prime} \omega^{\prime \lambda \omega}} \cdot\left(r_{1}^{\lambda} \mathrm{e}^{-\omega r_{1}} L_{\nu-\lambda-1}^{2 \lambda+1}\left(2 \omega r_{1}\right)\right) \mathrm{d} r_{1} \\
= & -A_{\nu^{\prime} \lambda^{\prime} \omega^{\prime} \omega^{\prime}}^{2 \lambda}\left(r_{2}^{\lambda} \mathrm{e}^{-\omega r_{2}} L_{\nu-\lambda-1}^{2 \lambda+1}\left(2 \omega r_{2}\right)\right) .
\end{aligned}
$$

Further integration over $r_{2}$ becomes relatively trivial.

Since

$$
\left(\frac{\mathrm{d}^{2}}{\mathrm{~d} r^{2}}+\frac{2}{r} \frac{\mathrm{d}}{\mathrm{d} r}-\frac{\lambda(\lambda+1)}{r^{2}}+\frac{2 \omega \nu}{r}-\omega^{2}\right)\left(r^{\lambda} \mathrm{e}^{-\omega r} L_{\nu-\lambda-1}^{2 \lambda+1}(2 \omega r)\right)=0
$$

we can readily find such operator pairs. Thus, when $A_{\nu^{\prime} \lambda^{\prime} \omega^{\prime}}^{\nu \omega}=u\left(r_{1}\right)$ for some general differentiable function $u$,

$$
\begin{aligned}
B_{\substack{\prime \\
v^{\prime} \lambda^{\prime} \omega^{\prime}}}^{\nu \lambda \omega}=2 u^{\prime}\left(r_{1}\right) & \frac{\mathrm{d}}{\mathrm{d} r_{1}}+u^{\prime \prime}\left(r_{1}\right)+\frac{2}{r_{1}} u^{\prime}\left(r_{1}\right) \\
& +\left(\left(\omega^{2}-\omega^{\prime 2}\right)+\frac{\lambda(\lambda+1)-\lambda^{\prime}\left(\lambda^{\prime}+1\right)}{r_{1}^{2}}+\frac{2\left(\omega^{\prime} \nu^{\prime}-\omega \nu\right)}{r_{1}}\right) u\left(r_{1}\right)
\end{aligned}
$$

and when $A_{\nu^{\prime} \lambda^{\prime} \omega^{\prime}}^{\nu \omega \omega}=v\left(r_{1}\right) \mathrm{d} / \mathrm{d} r_{1}$ for some general function $v$,

$$
\begin{gathered}
B_{\nu^{\prime} \lambda^{\prime} \omega^{\prime}}^{\nu \lambda \omega}=\left[v^{\prime \prime}\left(r_{1}\right)-\frac{2}{r_{1}} v^{\prime}\left(r_{1}\right)+\left(\frac{2+\lambda(\lambda+1)-\lambda^{\prime}\left(\lambda^{\prime}+1\right)}{r_{1}^{2}}+\left(\omega^{2}-\omega^{\prime 2}\right)\right.\right. \\
\left.\left.+\frac{2\left(\omega^{\prime} \nu^{\prime}-\omega \nu\right)}{r_{1}}\right) v\left(r_{1}\right)\right] \frac{\mathrm{d}}{\mathrm{d} r_{1}}+2\left(\frac{\lambda(\lambda+1)}{r_{1}^{2}}-\frac{2 \omega \nu}{r_{1}}+\omega^{2}\right) v^{\prime}\left(r_{1}\right) \\
+2\left(\frac{\omega \nu}{r_{1}^{2}}-\frac{\lambda(\lambda+1)}{r_{1}^{3}}\right) v\left(r_{1}\right) .
\end{gathered}
$$

Obviously we can combine the above two forms and the general result (6.3) still holds. For example, if we combine the expressions arising when we choose $u(r)$ equal to $r^{m}, r^{m+1}$, and $r^{m-1}$ and $v(r)$ equal to $r^{m}$ in such a way that the term in $\mathrm{d} / \mathrm{d} r_{1}$ vanishes, we are left with the following recursion relation for matrix elements of powers of $r$ :

$$
\begin{aligned}
\int_{0}^{\infty} r_{1}^{2} g_{\nu^{\prime} \lambda} \cdot\left(r_{1},\right. & \left.r_{2} ; \omega^{\prime}\right)\left(r_{1}^{l} \mathrm{e}^{-\omega r_{1}} L_{\nu-\lambda-1}^{2 \lambda+1}\left(2 \omega r_{1}\right)\right) \\
& \times\left(b_{+1} r_{1}^{m+1}+b_{0} r_{1}^{m}+b_{-1} r_{1}^{m-1}+b_{-2} r_{1}^{m-2}+b_{-3} r_{1}^{m-3}\right) \mathrm{d} r_{1} \\
= & -\left(a_{+1} r_{2}^{m+1}+a_{0} r_{2}^{m}+a_{-1} r_{2}^{m-1}+r_{2}^{m} \mathrm{~d} / \mathrm{d} r_{2}\right)\left(r_{2}^{\lambda} \mathrm{e}^{-\omega r_{2}} L_{\nu-\lambda-1}^{2 \lambda+1}\left(2 \omega r_{2}\right)\right)
\end{aligned}
$$


where

$$
\begin{aligned}
& a_{+1}=-\left(\omega^{2}-\omega^{\prime 2}\right) /(2 m+2) \quad a_{0}=-\left(\omega^{\prime} \nu^{\prime}-\omega \nu\right) / m \\
& a_{-1}=-\left[(m-1)(m-2)+\lambda(\lambda+1)-\lambda^{\prime}\left(\lambda^{\prime}+1\right)\right] /(2 m-2)
\end{aligned}
$$

and

$$
\begin{aligned}
& b_{+1}=\left(\omega^{2}-\omega^{\prime 2}\right) a_{+1} \quad b_{0}=2\left(\omega^{\prime} \nu^{\prime}-\omega \nu\right) a_{+1}+\left(\omega^{2}-\omega^{\prime 2}\right) a_{0} \\
& b_{-1}=2 m \omega^{2}+\left[(m+1)(m+2)+\lambda(\lambda+1)-\lambda^{\prime}\left(\lambda^{\prime}+1\right)\right] a_{+1}+2\left(\omega^{\prime} \nu^{\prime}-\omega \nu\right) a_{0} \\
& \quad+\left(\omega^{2}-\omega^{\prime 2}\right) a_{-1} \\
& b_{-2}=-2 \omega \nu(2 m-1)+\left[m(m+1)+\lambda(\lambda+1)-\lambda^{\prime}\left(\lambda^{\prime}+1\right)\right] a_{0}+2\left(\omega^{\prime} \nu^{\prime}-\omega \nu\right) a_{-1} \\
& b_{-3}=2(m-1) \lambda(\lambda+1)+\left[m(m+1)+\lambda(\lambda+1)-\lambda^{\prime}\left(\lambda^{\prime}+1\right)\right] a_{-1} .
\end{aligned}
$$

The coefficients listed above are written for general values of the parameters. For the non-relativistic SCGF, however, some simplification is immediately apparent since in that case $\omega \nu=\omega^{\prime} \nu^{\prime}=1 / a$ and both $a_{0}$ and $b_{0}$ vanish.

Another relation between matrix elements which follows immediately from (6.3) is a generalized orthogonality property of the radial CGFs. If we choose $u(r)=1$ we find

$$
\begin{aligned}
\int_{0}^{\infty}\left[r_{1}^{2}\left(\omega^{2}-\omega^{\prime 2}\right)+2\left(\omega^{\prime} \nu^{\prime}-\omega \nu\right) r_{1}+\lambda(\lambda+1)-\lambda^{\prime}\left(\lambda^{\prime}+1\right)\right] g_{\nu^{\prime} \lambda} \cdot\left(r_{1}, r_{2} ; \omega^{\prime}\right) \\
\quad \times\left(r_{1}^{\lambda} \mathrm{e}^{-\omega r_{1}} L_{\nu-\lambda-1}^{2 \lambda+1}\left(2 \omega r_{1}\right)\right) \mathrm{d} r_{1} \\
=-r_{2}^{\lambda} \mathrm{e}^{-\omega r_{2}} L_{\nu-\lambda-1}^{2 \lambda+1}\left(2 \omega r_{2}\right) .
\end{aligned}
$$

Thus in the non-relativistic case we have

$$
\begin{aligned}
& \left(n^{2}-\nu^{2}\right) /(a n \nu)^{2} \int_{0}^{\infty} r_{1}^{2} g_{l}\left(r_{1}, r_{2} ; \nu\right) R_{n l}\left(r_{1}\right) \mathrm{d} r_{1} \\
& =\left[l(l+1)-l^{\prime}\left(l^{\prime}+1\right)\right] \int_{0}^{\infty} g_{l^{\prime}}\left(r_{1}, r_{2} ; \nu\right) R_{n l}\left(r_{1}\right) \mathrm{d} r_{1}+2 m / \hbar^{2} R_{n l}\left(r_{2}\right)
\end{aligned}
$$

which, when $l=l^{\prime}$ reduces to the obvious orthogonality relation

$$
\left(n^{2}-\nu^{2}\right) /(a n \nu)^{2} \int_{0}^{\infty} r_{1}^{2} g_{1}\left(r_{1}, r_{2} ; \nu\right) R_{n l}\left(r_{1}\right) \mathrm{d} r_{1}=2 m / \hbar^{2} R_{n I}\left(r_{2}\right) .
$$

It is clear that any number of recursion relations for the matrix elements of the radial CGFs can be derived from (6.3), (6.5) and (6.6). Which will be of use will obviously depend on the specific calculations under consideration.

\section{Acknowledgments}

Research support by the Natural Sciences and Engineering Research Council of Canada is gratefully acknowledged. 


\section{Appendix 1. Solution of the generalized radial equation}

In this appendix we present a proof that the Sturmian form of the radial CGF is a solution of the defining differential equation. Indeed we show that the solution of

$$
\left[\frac{\mathrm{d}^{2}}{\mathrm{~d} r_{1}^{2}}+\frac{2}{r_{1}} \frac{\mathrm{d}}{\mathrm{d} r_{1}}-\frac{\lambda(\lambda+1)}{r_{1}^{2}}+\frac{2 \omega \nu}{r_{1}}-\omega^{2}\right] g_{\nu \lambda}\left(r_{1}, r_{2} ; \omega\right)=-\frac{\delta\left(r_{1}-r_{1}\right)}{r_{1} r_{2}}
$$

subject to the boundary conditions that

$$
\lim _{r_{1} \rightarrow 0} r_{1} g_{\nu \lambda}\left(r_{1}, r_{2} ; \omega\right)=0 ; r_{1} g_{\nu \lambda}\left(r_{2} ; \omega\right) \in L^{2}(\mathbb{R})
$$

and where $\lambda, \omega$, and $\nu$ are positive real numbers, is

$$
\begin{aligned}
g_{\nu \lambda}\left(r_{1}, r_{2} ; \omega\right) & \equiv(2 \omega)^{2 \lambda+1}\left(r_{1} r_{2}\right)^{\lambda} \mathrm{e}^{-\left(r_{1}+r_{2}\right) \omega} \\
& \times \sum_{k=0}^{\infty} \frac{k !}{\Gamma(2 \lambda+2+k)(\lambda+1+k-\nu)} L_{k}^{2 \lambda+1}\left(2 \omega r_{1}\right) L_{k}^{2 \lambda+1}\left(2 \omega r_{2}\right) .
\end{aligned}
$$

We will actually solve for the function

$$
f\left(r_{1}, r_{2}\right)=\left(r_{1} r_{2}\right)^{\lambda+1} g_{\nu \lambda}\left(r_{1}, r_{2} ; \omega\right)
$$

which satisfies

$$
\left[r_{1} \frac{\mathrm{d}^{2}}{\mathrm{~d} r_{1}^{2}}-2 \lambda \frac{\mathrm{d}}{\mathrm{d} r_{1}}+2 \omega \nu-r_{1} \omega^{2}\right] f\left(r_{1}, r_{2}\right)=-r_{1}\left(r_{1} r_{2}\right)^{\lambda} \delta\left(r_{1}-r_{2}\right) .
$$

Now the double Laplace transform of $f$ is

$$
S_{\nu \lambda}\left(p_{1}, p_{2} ; \omega\right)=\int_{0}^{\infty} \int_{0}^{\infty} \mathrm{e}^{-p_{1} r_{1}-p_{2} r_{2}} f\left(r_{1}, r_{2}\right) \mathrm{d} r_{1} \mathrm{~d} r_{2}
$$

and satisfies the transformed equation

$$
\left(\left(p_{1}^{2}-\omega^{2}\right) \frac{\mathrm{d}}{\mathrm{d} p_{1}}+2 p_{1}(\lambda+1)-2 \omega \nu\right) S_{\nu \lambda}\left(p_{1}, p_{2} ; \omega\right)=\frac{\Gamma(2 \lambda+2)}{\left(p_{1}+p_{2}\right)^{2 \lambda+2}}
$$

where we have used the fact that $f\left(0, r_{2}\right)=0$. Hence, letting

$$
E\left(p_{1}, p_{2}\right)=\frac{\left(p_{1}+p_{2}\right)^{2 \lambda+2}}{\Gamma(2 \lambda+2)} S_{\nu \lambda}\left(p_{1}, p_{2} ; \omega\right)
$$

so that

$$
\left(\left(p_{1}^{2}-\omega^{2}\right) \frac{\mathrm{d}}{\mathrm{d} p_{1}}+\frac{(2 \lambda+2)}{\left(p_{1}+p_{2}\right)}\left(p_{1} p_{2}+\omega^{2}\right)-2 \omega \nu\right) E\left(p_{1}, p_{2}\right)=1
$$

and then making the change of variables

$$
p_{1} \mapsto z=-1 / \omega\left(p_{1}+\omega\right)\left(p_{2}-\omega\right) / 2\left(p_{1}+p_{2}\right)
$$

gives finally

$$
\left(z(1-z) \frac{\mathrm{d}}{\mathrm{d} z}+(\lambda+1)(1-2 z)-\nu\right) E(z)=1 /(2 \omega) .
$$


The solution to this last equation is easily found as a series in $z$, provided that $\nu-\lambda-1$ is not an integer (which would not be true if we were considering the reduced Coulomb Green function [2]). We find a particular solution to (A1.11)

$$
E_{\mathrm{p}}(z)=1 / \omega_{2} F_{1}(2 \lambda+2,1 ; \lambda+2-\nu ; z)
$$

and a solution of the corresponding homogeneous equation

$$
E_{\mathrm{h}}(z)=N z^{\nu-\lambda-1}(z-1)^{-\nu-\lambda-1}
$$

where $N$ is an arbitrary constant. Invoking a well-known transformation of the hypergeometric function, we arrive at the general solution to (A1.11):

$$
\begin{gathered}
E(z)=1 / \omega(1-z)^{-2 \lambda-2}{ }_{2} F_{1}\left(2 \lambda+2, \lambda+1-\nu ; \lambda+2-\nu ; \frac{z}{z-1}\right) / 2(\lambda-\nu+1) \\
+N z^{\nu-\lambda-1}(z-1)^{-\nu-\lambda-1} .
\end{gathered}
$$

The double Laplace transform of $f$ is therefore given by

$S_{\nu \lambda}\left(p_{1}, p_{2} ; \omega\right)$

$$
\begin{aligned}
= & (2 \omega)^{2 \lambda+1} \frac{\Gamma(2 \lambda+2)}{(\lambda-\nu+1)}\left[\left(p_{1}+\omega\right)\left(p_{2}+\omega\right)\right]^{-2 \lambda-2} \\
& \times{ }_{2} F_{1}\left[2 \lambda+2, \lambda+1-\nu ; \lambda+2-\nu ;\left(\frac{p_{1}-\omega}{p_{1}+\omega}\right)\left(\frac{p_{2}-\omega}{p_{2}+\omega}\right)\right] \\
& +N\left[\left(p_{1}-\omega\right)\left(p_{2}-\omega\right)\right]^{\nu-\lambda-1}\left[\left(p_{1}+\omega\right)\left(p_{2}+\omega\right)\right]^{-\nu-\lambda-1} .
\end{aligned}
$$

Now, in terms of $p_{1}, S$ has a singularity of largest real part at $p_{1}=\omega$ if $N \neq 0$. As a consequence of a theorem on the asymptotic forms of functions and their Laplace transforms [23] we can infer that

$$
g_{\nu \lambda}\left(r_{1}, r_{2} ; \omega\right) \approx N r_{1}^{\lambda-\nu} \mathrm{e}^{r_{1} \omega} \quad \text { as } r_{1} \rightarrow \infty
$$

which is at variance with the boundary conditions we have imposed. If $\nu>\lambda+1$ there is no singularity, but since we are looking for a solution valid for all real $\nu$ and continuous in $\nu$, we deduce that $N=0$. Hence

$S_{\nu \wedge}\left(p_{1}, p_{2} ; \omega\right)$

$$
\begin{aligned}
= & (2 \omega)^{2 \lambda+1}\left[\left(p_{1}+\omega\right)\left(p_{2}+\omega\right)\right]^{-2 \lambda-2} \sum_{k=0}^{\infty} \frac{\Gamma(2 \lambda+2+k)}{(\lambda+1+k-\nu) k !} \\
& \times\left(\frac{p_{1}-\omega}{p_{1}+\omega}\right)^{k}\left(\frac{p_{2}-\omega}{p_{2}+\omega}\right)^{k} .
\end{aligned}
$$

The inverse double Laplace transform of $S$ is easily obtained, since $S$ itself is an infinite sum of products of single Laplace transforms. We note that

$$
\begin{aligned}
& \mathscr{L}\left[\mathrm{e}^{-r \sigma} r^{\alpha} L_{n}^{\alpha}(2 r \sigma)\right](p)=\frac{\Gamma(\alpha+n+1)(p-\sigma)^{n}}{n !(p+\sigma)^{n+\alpha+1}} \\
& \mathscr{R}(\alpha)>-1, \mathscr{R}(p)>-\sigma
\end{aligned}
$$

so that performing the inverse double Laplace transformation we get

$$
\begin{aligned}
& f\left(r_{1}, r_{2}\right)=(2 \omega)^{2 \lambda+1}\left(r_{1} r_{2}\right)^{2 \lambda+1} \mathrm{e}^{-\left(r_{1}+r_{2}\right) \omega} \\
& \times \sum_{k=0}^{\infty} \frac{k !}{\Gamma(2 \lambda+2+k)(\lambda+1+k-\nu)} L_{k}^{2 \lambda+1}\left(2 \omega r_{1}\right) L_{k}^{2 \lambda+1}\left(2 \omega r_{2}\right)
\end{aligned}
$$

from which the main result (A1.3) follows immediately. 
It is useful to note the points at which the boundary conditions have been used: (i) in the derivation of the transformed equation (A1.7); (ii) in the deduction that the homogeneous part of the solution to (A1.11) is unacceptable. This treatment of the Laplace-transformed defining equation for the radial Green function, especially the use of the transformation (A1.10) has also been noted by Hill and Huxtable [24], Talukdar et al [25] and in [4]. The derivation of the inverse Laplace transform is, it appears, new.

Finally we present a representation of the Dirac delta function which will be of use later on. We have exhibited explicitly the solution to (A1.1); furthermore, we can easily calculate the effect of the differential operator on this solution using results obtained in [1]. Thus, substituting (A1.3) directly into (A1.1) leads to the following interesting formula:

$$
\alpha^{2 \lambda+2}(x y)^{\lambda} \mathrm{e}^{-\alpha(x+y) / 2} \sum_{k=0}^{\infty} \frac{k !}{\bar{\Gamma}(2 \lambda+2+k)} L_{k}^{2 \lambda+1}(\alpha x) L_{k}^{2 \lambda+1}(\alpha y)=\delta(x-y) / \sqrt{x y} .
$$

This can be thought of as either a representation of the delta function, or as an expression for the infinite sum of Laguerre polynomials or as a direct proof of the completeness of the Sturmian wavefunctions. Formula (A1.19) is used repeatedly in this and the following paper [2].

\section{Appendix 2. Single variable differential properties}

In order to calculate the off-diagonal terms of the radial DCGF (section 3) as well as the recursion relations for the general radial CGF (section 4$)$ we find it necessary to calculate both $\left[\lambda\left(\mathrm{d} / \mathrm{d} r_{1}+(\lambda+1) / r_{1}\right)-\omega \nu\right] g_{i \lambda}\left(r_{1}, r_{2} ; \omega\right)$ and $\left[\lambda\left(\mathrm{d} / \mathrm{d} r_{1}-(\lambda-1) / r_{1}\right)+\right.$ $\omega \nu] g_{\nu \lambda-1}\left(r_{1}, r_{2} ; \omega\right)$. This we will do in this appendix. As our starting point, since ([1], equation 3.29)

$$
\begin{aligned}
& {\left[\lambda\left(\frac{\mathrm{d}}{\mathrm{d} r}+\frac{\lambda+1}{r}\right)-\omega \nu\right]\left(r^{\lambda} \mathrm{e}^{-r \omega} L_{k}^{2 \lambda+1}(2 \omega r)\right)} \\
& =\omega(k+\lambda+1-\nu) r^{\lambda} \mathrm{e}^{-r \omega} L_{k}^{2 \lambda+1}(2 \omega r) \\
& \quad+(k+1)(2 \lambda+k+1) / 2 r^{\lambda-1} \mathrm{e}^{-r \omega} L_{k+1}^{2 \lambda-1}(2 \omega r)
\end{aligned}
$$

we find

$$
\begin{aligned}
& {\left[\lambda\left(\frac{\mathrm{d}}{\mathrm{d} r_{1}}+\frac{\lambda+1}{r_{1}}\right)-\omega \nu\right] g_{\nu \lambda}\left(r_{1}, r_{2} ; \omega\right) } \\
&=(2 \omega)^{2 \lambda+1}\left(r_{1} r_{2}\right)^{\lambda} \mathrm{e}^{-\left(r_{1}+r_{2}\right) \omega}\left(\omega \sum_{k=0}^{\infty} \frac{k !}{\Gamma(2 \lambda+2+k)} L_{k}^{2 \lambda+1}\left(2 \omega r_{1}\right) L_{k}^{2 \lambda+1}\left(2 \omega r_{2}\right)\right. \\
&\left.+1 /\left(2 r_{1}\right) \sum_{k=0}^{\infty} \frac{(k+1) !}{\Gamma(2 \lambda+1+k)(\lambda+1+k-\nu)} L_{k+1}^{2 \lambda-1}\left(2 \omega r_{1}\right) L_{k}^{2 \lambda+1}\left(2 \omega r_{2}\right)\right) \\
&=\frac{1}{2}\left[\delta\left(r_{1}-r_{2}\right) / \sqrt{r_{1} r_{2}}+(2 \omega)^{2 \lambda+1} r_{1}^{\lambda-1} r_{2}^{\lambda} \mathrm{e}^{\left.-1 r_{1}+r_{2}\right) \omega}\right. \\
&\left.\times \sum_{k=0}^{\infty} \frac{(k+1) !}{\Gamma(2 \lambda+1+k)(\lambda+1+k-\nu)} L_{k+1}^{2 \lambda-1}\left(2 \omega r_{1}\right) L_{k}^{2 \lambda+1}\left(2 \omega r_{2}\right)\right]
\end{aligned}
$$

where in the last step we have used (A1.19). 
The second calculation is performed in a similar manner. Since ([1], equation 3.30)

$$
\begin{aligned}
{\left[\lambda\left(\frac{\mathrm{d}}{\mathrm{d} r}-\frac{\lambda-1}{r}\right)+\omega \nu\right]\left(r^{\lambda-1} \mathrm{e}^{-r \omega} L_{k}^{2 \lambda-1}(2 \omega r)\right) } \\
=-\omega(k+\lambda-\nu) r^{\lambda-1} \mathrm{e}^{-r \omega} L_{k}^{2 \lambda-1}(2 \omega r) \\
-2 \omega^{2}\left\{1-\delta_{k 0}\right\} r^{\lambda} \mathrm{e}^{-r \omega} L_{k-1}^{2 \lambda+1}(2 \omega r)
\end{aligned}
$$

we now deduce that

$$
\begin{aligned}
{\left[\lambda\left(\frac{\mathrm{d}}{\mathrm{d} r_{1}}-\frac{\lambda-1}{r_{1}}\right)\right.} & +\omega \nu] g_{\nu \lambda-1}\left(r_{1}, r_{2} ; \omega\right) \\
= & -\frac{1}{2}\left[\delta\left(r_{1}-r_{2}\right) / \sqrt{r_{1} r_{2}}+(2 \omega)^{2 \lambda+1} r_{1}^{\lambda} r_{2}^{\lambda-1} \mathrm{e}^{-\left(r_{1}+r_{2}\right) \omega}\right. \\
& \left.\times \sum_{k=0}^{\infty} \frac{(k+1) !}{\Gamma(2 \lambda+1+k)(\lambda+1+k-\nu)} L_{k}^{2 \lambda+1}\left(2 \omega r_{1}\right) L_{k+1}^{2 \lambda-1}\left(2 \omega r_{2}\right)\right] .
\end{aligned}
$$

The differential relations expressed by (A2.2) and (A2.4) constitute the main results of this appendix.

\section{Appendix 3. Alternate derivation of recursion relations for the radial Green functions}

We have shown in appendix 1 that the double Laplace transform, $\mathscr{L}\left[\left(r_{1} r_{2}\right)^{\lambda+1} g_{\nu \lambda}\left(r_{1}, r_{2} ; \omega\right)\right]\left(p_{1}, p_{2}\right)$, is

$$
\begin{aligned}
S_{\nu \lambda}\left(p_{1}, p_{2} ; \omega\right) & =(2 \omega)^{2 \lambda+1}\left[\left(p_{1}+\omega\right)\left(p_{2}+\omega\right)\right]^{-2 \lambda-2} \\
& \times \sum_{k=0}^{\infty} \frac{\Gamma(2 \lambda+2+k)}{(\lambda+1+k-\nu) k !}\left(\frac{p_{1}-\omega}{p_{1}+\omega}\right)^{k}\left(\frac{p_{2}-\omega}{p_{2}+\omega}\right)^{k}
\end{aligned}
$$

and satisfies

$\left(\left(p_{1}^{2}-\omega^{2}\right) \frac{\mathrm{d}}{\mathrm{d} p_{1}}+2 p_{1}(\lambda+1)-2 \omega \nu\right) S_{\nu \lambda}\left(p_{1}, p_{2} ; \omega\right)=\frac{\Gamma(2 \lambda+2)}{\left(p_{\mathrm{t}}+p_{2}\right)^{2 \lambda+2}}$.

Now

$$
\begin{aligned}
& \sum_{k=0}^{\infty} \frac{\Gamma(2 \lambda+k)}{(\lambda+k-\nu) k !} x^{k} \\
& =\frac{\Gamma(2 \lambda)}{(\lambda-\nu)}(1-x)^{-2 \lambda}+\frac{\Gamma(2 \lambda+1)}{\left(\lambda^{2}-\nu^{2}\right)} x(1-x)^{-2 \lambda-1} \\
& \quad-x /\left(\lambda^{2}-\nu^{2}\right) \sum_{k=0}^{\infty} \frac{\Gamma(2 \lambda+2+k)}{(\lambda+k+1-\nu) k !} x^{k}
\end{aligned}
$$

so that

$$
\begin{aligned}
(2 \omega)^{2}\left(\lambda^{2}-\nu^{2}\right) & S_{\nu \lambda-1}\left(p_{1}, p_{2} ; \omega\right) \\
= & (2 \omega)(\lambda+\nu) \Gamma(2 \lambda)\left(p_{1}+p_{2}\right)^{-2 \lambda}+\Gamma(2 \lambda+1)\left(p_{1}-\omega\right)\left(p_{2}-\omega\right)\left(p_{1}+p_{2}\right)^{-2 \lambda-1} \\
& -\left(p_{1}^{2}-\omega^{2}\right)\left(p_{2}^{2}-\omega^{2}\right) S_{\nu \lambda}\left(p_{2}, p_{2} ; \omega\right) .
\end{aligned}
$$

This is the fundamental relation which we will use to derive the raising and lowering operators for $\lambda$ in the Green functions. 
We begin our analysis by differentiating (A3.4) with respect to $p_{1}$, using (A3.2) to show that

$$
\begin{aligned}
2 \omega^{2}\left(\lambda^{2}-\nu^{2}\right) & \frac{\mathrm{d}}{\mathrm{d} p_{1}} S_{\nu \lambda-1}\left(p_{1}, p_{2} ; \omega\right) \\
= & -\Gamma(2 \lambda+1)\left(\lambda p_{2}+\omega \nu\right)\left(p_{1}+p_{2}\right)^{-2 \lambda-1} \\
& +\left(\lambda p_{1}-\omega \nu\right)\left(p_{2}^{2}-\omega^{2}\right) S_{\nu_{\lambda}}\left(p_{1}, p_{2} ; \omega\right)
\end{aligned}
$$

from which, again using (3.2) (for $p_{2}$ ), it follows that

$$
\begin{aligned}
\omega^{2}\left(\lambda^{2}-\omega^{2}\right) & \frac{\mathrm{d}}{\mathrm{d} p_{1}} \frac{\mathrm{d}}{\mathrm{d} p_{2}} S_{\nu \lambda-1}\left(p_{1}, p_{2} ; \omega\right) \\
& =\lambda^{2} \Gamma(2 \lambda+1)\left(p_{1}+p_{2}\right)^{-2 \lambda-1}-\left(\lambda p_{1}-\omega\right)\left(\lambda p_{2}-\omega\right) S_{\nu \lambda}\left(p_{1}, p_{2} ; \omega\right) .
\end{aligned}
$$

This is our $\lambda$ lowering equation.

Our next relation, which is obtained in much the same manner, and using the previous three equations, is

$$
\begin{aligned}
\left(\left(\lambda p_{1}+\omega \nu\right)\right. & \left.\frac{\mathrm{d}}{\mathrm{d} p_{1}}+2 \lambda^{2}\right)\left(\left(\lambda p_{2}+\omega \nu\right) \frac{\mathrm{d}}{\mathrm{d} p_{2}}+2 \lambda^{2}\right) S_{\nu \lambda-1}\left(p_{1}, p_{2} ; \omega\right) \\
& =\lambda^{2} \Gamma(2 \lambda+1)\left(p_{1}+p_{2}\right)^{-2 \lambda-1}-\omega^{2}\left(\lambda^{2}-\nu^{2}\right) S_{\nu \lambda}\left(p_{1}, p_{2} ; \omega\right)
\end{aligned}
$$

This is our $\lambda$ raising equation.

Finally we can easily derive from the previous results a mixed raising and lowering operator:

$\left(\left(\lambda p_{1}+\omega \nu\right) \frac{\mathrm{d}}{\mathrm{d} p_{1}}+2 \lambda^{2}\right) \frac{\mathrm{d}}{\mathrm{d} p_{2}} S_{\nu \lambda-1}\left(p_{1}, p_{2} ; \omega\right)=-\left(\lambda p_{2}-\omega \nu\right) S_{\nu \lambda}\left(p_{1}, p_{2} ; \omega\right)$

$\lambda$ raising and lowering operators for the Green function itself are obtained quite simply by taking the inverse double Laplace transform of the relevant equations given above. The inverse of (A3.6) is the $\lambda$ lowering operator for $g$, (5.4), while the inverse of (A3.7) is a $\lambda$ raising operator, (5.5). Finally, the mixed raising and lowering operator (5.3) is obtained as the inverse of (A3.8).

\section{Appendix 4. Notation and special functions}

Hypergeometric functions

$$
\begin{aligned}
& (\alpha)_{n}=\Gamma(\alpha+n) / \Gamma(\alpha) \\
& { }_{1} F_{1}(\alpha ; \beta ; x)=\sum \frac{(\alpha)_{n}}{(\beta)_{n}} \frac{x^{n}}{n !} \\
& { }_{2} F_{1}(\alpha, \beta ; \gamma ; x)=\sum \frac{(\alpha)_{n}(\beta)_{n}}{(\gamma)_{n}} \frac{x^{n}}{n !} \\
& { }_{p} F_{q}\left(\alpha_{1}, \ldots ; \beta_{1}, \ldots ; x\right)=\sum \frac{\left(\alpha_{1}\right)_{n} \ldots\left(\alpha_{p}\right)_{n}}{\left(\beta_{1}\right)_{n} \ldots\left(\beta_{q}\right)_{n}} \frac{x^{n}}{n !} \\
& F_{1}\left(\alpha ; \beta_{1}, \beta_{2} ; \gamma ; x, y\right)=\sum \frac{(\alpha)_{n+m}\left(\beta_{1}\right)_{n}\left(\beta_{2}\right)_{m}}{(\gamma)_{n+m}} \frac{x^{n}}{n !} \frac{y^{m}}{m !} \\
& F_{2}\left(\alpha ; \beta_{1}, \beta_{2} ; \gamma_{1}, \gamma_{2} ; x, y\right)=\sum \frac{(\alpha)_{n+m}\left(\beta_{1}\right)_{n}\left(\beta_{2}\right)_{m}}{\left(\gamma_{1}\right)_{n}\left(\gamma_{2}\right)_{m}} \frac{x^{n}}{n !} \frac{y^{m}}{m !}
\end{aligned}
$$


Laguerre polynomials

$$
\begin{aligned}
& L_{n}^{\alpha}(x)=\frac{\Gamma(\alpha+1+n)}{\Gamma(\alpha+1) n !}{ }_{1} F_{1}(-n ; \alpha+1 ; x) \\
& (n+1) L_{n+1}^{\alpha}(x)+(x-\alpha-2 n-1) L_{n}^{\alpha}(x)+(n+\alpha) L_{n-1}^{\alpha}(x)=0 .
\end{aligned}
$$

Whittaker functions

$$
\begin{aligned}
& M_{\nu, \lambda}(x)=x^{\lambda+1 / 2} \mathrm{e}^{-x / 2}{ }_{1} F_{1}(\lambda+1 / 2-\nu ; 2 \lambda+1 ; x) \\
& =x^{\lambda+1 / 2} \mathrm{e}^{+x / 2}{ }_{1} F_{1}(\lambda+1 / 2+\nu ; 2 \lambda+1 ;-x) \\
& W_{\nu, \lambda}(x)=\frac{\Gamma(-2 \lambda)}{\Gamma(1 / 2-\lambda-\nu)} M_{\nu, \lambda}(x)+\frac{\Gamma(2 \lambda)}{\Gamma(1 / 2+\lambda-\nu)} M_{\nu,-\lambda}(x) \\
& (1+2 \lambda) \frac{\mathrm{d}}{\mathrm{d} x} M_{\nu, \lambda}(x) \\
& =\left[(1+2 \lambda)^{2} / 2 x-\nu\right] M_{\nu, \lambda}(x)-\left[\nu^{2}-(1 / 2+\lambda / 2)^{2}\right] /(2+2 \lambda) \\
& \times(1+2 \lambda) M_{v, \lambda+1}(x) \\
& (1-2 \lambda) \frac{\mathrm{d}}{\mathrm{d} x} M_{\nu, \lambda}(x)=\left[(1-2 \lambda)^{2} / 2 x-\nu\right] M_{\nu, \lambda}(x)-(2 \lambda+1)(2 \lambda) M_{\nu, \lambda-1}(x) \\
& (1+2 \lambda) \frac{\mathrm{d}}{\mathrm{d} x} W_{\nu, \lambda}(x)=\left[(1+2 \lambda)^{2} / 2 x-\nu\right] W_{\nu, \lambda}(x)+[\nu-1 / 2-\lambda] W_{\nu, \lambda+1}(x) \\
& (1-2 \lambda) \frac{\mathrm{d}}{\mathrm{d} x} W_{\nu_{0},}(x)=\left[(1-2 \lambda)^{2} / 2 x-\nu\right] W_{\nu, \lambda}(x)+[\nu-1 / 2+\lambda] W_{\nu, \lambda-1}(x) .
\end{aligned}
$$

Bessel functions

$$
\begin{aligned}
& J_{\nu}(z)=\sum_{k=0}^{\infty} \frac{(-1)^{k}(z / 2)^{\nu+2 k}}{\Gamma(k+1) \Gamma(k+\nu+1)} \\
& I_{\nu}(z)=\sum_{k=0}^{\infty} \frac{(z / 2)^{\nu+2 k}}{\Gamma(k+1) \Gamma(k+\nu+1)} .
\end{aligned}
$$

Dirac matrices

$$
\begin{aligned}
& \boldsymbol{\alpha}=\left(\begin{array}{ll}
0 & \boldsymbol{\sigma} \\
\boldsymbol{\sigma} & 0
\end{array}\right) \quad \beta=\left(\begin{array}{rr}
1 & 0 \\
0 & -1
\end{array}\right) \\
& \boldsymbol{\sigma}=\left(\left(\begin{array}{ll}
0 & 1 \\
1 & 0
\end{array}\right),\left(\begin{array}{rr}
0 & -i \\
i & 0
\end{array}\right),\left(\begin{array}{rr}
1 & 0 \\
0 & -1
\end{array}\right)\right) \\
& \mathbf{\Sigma}=\left(\begin{array}{ll}
\boldsymbol{\sigma} & 0 \\
0 & \boldsymbol{\sigma}
\end{array}\right) .
\end{aligned}
$$




\section{References}

[1] Swainson R A and Drake G W F 1990 J. Phys. A: Math. Gen. 23 to be published

[2] Swainson R A and Drake G W F 1990 J. Phys. A: Math. Gen. to be submitted

[3] Edmonds A R 1957 Angular Momentum in Quantum Mechnics (Princeton, NJ: Princeton University Press)

[4] Swainson R A 1988 PhD Thesis University of Windsor

[5] Meixner J 1933 Math. Zeit. 36677

[6] Wichmann E H and Woo C H 1961 J. Math. Phys. 2178

[7] Hostler L 1964 J. Math. Phys. 5591

[8] Schwinger J 1964 J. Math. Phys. 51606

[9] Hostler L 1970 J. Math. Phys. 112966

[10] Garcia-Bondia J M 1984 Phys. Rev. A 30691

[11] Chetouani L and Hamman T F 1987 J. Math. Phys. 28598

[12] Wichmann E H and Kroll N M 1956 Phys. Rev. 101843

[13] Martin P C and Glauber R J 1958 Phys. Rev. 1091307

[14] Zapryagaev S A and Manakov N L 1976 Sov. J. Nucl. Phys. 23482

[15] Granovskii Y I and Nechet VI 1974 Theor. Math. Phys. 18185

[16] Infeld L 1941 Phys. Rev. 59737

[17] Maquet A 1977 Phys. Rev. A 151088

[18] Lebedev N N 1972 Special Functions and their Applications (New York: Dover)

[19] Swainson R A and Drake G W F 1988 Nucl. Instrum. Methods B 31115

[20] Buchholz H 1953 Die Konfluente Hypergeometrische Funktion (Berlin: Springer)

[21] Hylton D J 1984 J. Math. Phys. 251125

[22] Johnson B R and Hirschfelder J O 1979 J. Math. Phys. 202484

[23] Doetsch G 1961 Guide to the Applications of Laplace Transforms (New York: Van Nostrand)

[24] Hill R N and Huxtable B D 1982 J. Math. Phys. 232365

[25] Talukdar B, Laha U and Bhattaru S R 1985 J. Phys. A: Math. Gen. 18 L359 